NPI MSU $-99-10 / 568$

\title{
Finslerian Spaces Possessing Local Relativistic Symmetry
}

\author{
G.Yu. Bogoslovsky \\ Institute of Nuclear Physics, Moscow State University \\ 119899, Moscow, Russia \\ E-mail: bogoslov@theory.npi.msu.su \\ H.F. Goenner \\ Institute for Theoretical Physics, University of Göttingen \\ Bunsenstr.9, D-37073, Göttingen, Germany \\ E-mail: goenner@theorie.physik.uni-goettingen.de
}

\begin{abstract}
It is shown that the problem of a possible violation of the Lorentz transformations at Lorentz factors $\gamma>5 \times 10^{10}$, indicated by the situation which has developed in the physics of ultra-high energy cosmic rays (the absence of the GZK cutoff), has a nontrivial solution. Its essence consists in the discovery of the so-called generalized Lorentz transformations which seem to correctly link the inertial reference frames at any values of $\gamma$. Like the usual Lorentz transformations, the generalized ones are linear, possess group properties and lead to the Einstein law of addition of 3-velocities. However, their geometric meaning turns out to be different: they serve as relativistic symmetry transformations of a flat anisotropic Finslerian event space rather than of Minkowski space. Consideration is given to two types of Finsler spaces which generalize locally isotropic Riemannian space-time of relativity theory, e.g. Finsler spaces with a partially and entirely broken local 3D isotropy. The investigation advances arguments for the corresponding generalization of the theory of fundamental interactions and for a specific search for physical effects due to local anisotropy of space-time.
\end{abstract}

24/03/99 


\section{INTRODUCTION}

At present, apart from general relativity theory (GR), there exist a number of alternative metric theories of gravitation. They all employ the Riemannian geometric model of spacetime borrowed from GR, and differ only by the field equations which describe the selfconsistent dynamics of space-time and matter. The cosmological models based on such theories differ accordingly. Common to them, however, is the fact that space-time, being Riemannian and, consequently, locally isotropic, preserves its local isotropy during the evolution of the Universe.

Although, as it would seem, there is no reason to question the local isotropy of space (the more so as no violation of the law of angular momentum conservation has yet been revealed), there are some indirect indications that in our epoch space-time, on the average, has a weak relic local anisotropy, and that it therefore should be described by Finsler geometry [1] rather than by Riemann geometry. A strong local anisotropy of space-time might have occured at an early stage in the evolution of the Universe as a result of hightemperature phase transitions in its geometric structure, caused by a breaking of higher gauge symmetries and by the appearance of massive elementary particles. If this was the case, it is natural to assume that the local anisotropy of space decreased to its present low level $\left(<10^{-10}\right)$ due to the expansion of the Universe.

The existence of a local anisotropy of space-time is indirectly indicated by the following facts: (i) a breaking of the discrete space-time symmetries in weak interactions; (ii) an anisotropy of the relic background radiation filling the Universe; and (iii) the absence [2] of the effect of cutoff of the spectrum of primary ultra-high energy cosmic protons, i.e. of the so-called GZK cutoff $[3,4]$.

By a strict local isotropy of Riemannian space-time we imply that, at each point, its tangent space is Minkowski space't the isotropic event space of special relativity theory (SR). In Galilean coordinates, the pseudo-Euclidean metric is of the form $d s^{2}=d x_{0}^{2}-d \boldsymbol{x}^{2}$. As under the discrete transformations: $x_{0} \rightarrow-x_{0}, x_{\alpha} \rightarrow-x_{\alpha}$, this metric is invariant under the continuous transformations belonging to the 10-parameter inhomogeneous Lorentz or Poincaré group.]

From the mathematical point of view, the presence of the Poincaré group as a group of relativistic symmetry (isometry group) of the event space is the necessary and sufficient condition for it to be Minkowski space. Therefore, if the Poincaré symmetry turns out to be only approximate, and if the exact transformations of relativistic symmetry realized in nature are some "generalized Lorentz transformations" imbedded into another group, then the event space has a geometry different from that of Minkowski space - even at the level of SR.

The idea of a possible violation of the usual Lorentz transformations at Lorentz factors $\gamma>5 \times 10^{10}$, and of a corresponding generalization of the relativistic theories was suggested first in [5,6]. Its motivation rested on a discrepancy, assumed at the time, between

\footnotetext{
${ }^{1}$ The name Minkowski space is used here in the usual sense, i.e. for a 4-dimensional, pseudo-Euclidean, flat Riemannian space. In contrast, Rund [1] has used it for a flat Finsler space.

${ }^{2}$ Four parameters correspond to space-time translations, three to 3D rotations, and another three to Lorentz boosts.
} 
the theoretical predictions $[3,4]$ and the experimental data [7] relating to the behaviour of the spectrum of primary ultra-high energy cosmic protons. If the usual Lorentz transformations would correctly link inertial frames at relative velocities very close to the velocity of light, then, in the case of uniformly distributed sources, the energy spectrum of primary cosmic protons should show a cutoff (due to inelastic collisions of the protons with cosmic background radiation photons) at proton energies $\sim 5 \times 10^{19} \mathrm{eV}$. However, as now has been firmly established, such a prediction is at variance with present experimental data ${ }^{\text {J }}$.

Apart from the violation of the Lorentz transformations, there exist also other possible causes of the absence of the GZK cutoff [9]. Nevertheless, the assumption that the inertial frames could be linked by some "generalized Lorentz transformations" markedly different from the usual Lorentz transformations only at relative velocities extremely close to the velocity of light, remains valid. Moreover, general considerations make it possible to find the required transformations in an explicit form. There exists an 8-parameter group of relativistic symmetry obviously different from the Poincarè group [10]. Along with spacetime translations and the "generalized Lorentz transformations" (three parameters), the group includes only a 1-parameter subgroup of rotations of 3D space about some preferred direction. Since, as it turned out, such an 8-parameter group allows for a geometric invariant in the form of a flat Finsler metric generalizing the Minkowski metric of SR, the door is opened to a nontrivial generalization of relativity theory [11-13].

Although any relativistic theory is constructed from the requirement of invariance of its equations under the Poincaré group, soon after the creation of SR, the authors of [14,15] demonstrated invariance of the electrodynamic equations not only under Poincaré group but also under the 15-parameter conformal group. This group incorporates both linear and nonlinear transformations of event coordinates [16]. In terms of SR, the nonlinear transformations are of no interest since they link noninertial frames. A full classification of the subgroups of linear transformations of the conformal group has not yet been carried out. From the very outset, however, it was known that one of the linear subgroups of the conformal group is the Poincaré group.

In the next section it will be shown that the above-mentioned 8-parameter group is another linear subgroup of the conformal group. It likewise leads to Einstein's law of addition of 3-velocities. Therefore, if relativistic physics could have been developed already during a cosmological epoch with a sufficiently large local anisotropy of space and a flagrant violation of the conservation law of total angular momentum for any closed physical system, then the 8-parameter linear subgroup of the conformal group rather than the Poincaré subgroup might have been chosen as a group of relativistic symmetry. In order to empirically test such a choice in our epoch, very specific experiments are required since the rate of change of the total angular momentum is the lower, the smaller the magnitude of local space-time anisotropy will be. This signifies that non-conservation of angular momentum may primarily be manifested in processes for which the interaction time is long enough.

\footnotetext{
${ }^{3}$ In connection with this situation S. Coleman and S. L. Glashow [8] argue that possible departures from strict Lorentz invariance can affect elementary-particle kinematics so as to suppress or forbid inelastic collisions of cosmic-ray nucleons with background photons.

${ }^{4}$ i.e. the group whose invariant is the Finslerian metric describing a flat anisotropic event space
} 
When speaking of a flat locally anisotropic event space, we implied a space which, while not being symmetric with respect to arbitrary 3D rotations, is still symmetric with respect to rotations around some preferred direction and therefore will be referred to as a space with a partially broken 3D isotropy. Although as one of the consequences of this breaking of symmetry a violation of the conservation law of total angular momentum results, this is not inconsistent, but permissible from the point of view of physics. A demonstration is given by the analysis [17] of the corresponding generalized Dirac equation with the existence of stable massive fermions. Of physically interest seems also a model of space-time $[18,19]$ with an entirely broken local isotropy corresponding to a 7 -parameter inhomogeneous group of relativistic symmetry.

In section 2, we present a model of a flat Finsler space with a partially broken local rotational symmetry and sketch the induced point mechanics. Section 3 introduces a flat Finsler space with a totally broken local rotational symmetry. Here, the second simplest type of line elements for Finsler spaces already mentioned by Riemann [20] appears. Properties of the isometry group and some physical consequences of this model are discussed in sections 4 and 5. In both models the conformal structure of SR is preserved. Finally, section 6 briefly reviews a generalization to curved Finsler space, eventually needed for an interpretation of recent observational discoveries in astrophysics.

\section{A RELATIVISTICALLY SYMMETRIC FINSLERIAN SPACE-TIME WITH PARTIALLY BROKEN 3D ISOTROPY}

\subsection{Introducing the model}

In order to arrive at a viable Finslerian model of space-time, for the sake of simplicity we first confine ourselves to a two-dimensional space and show that it is possible to generalize the Lorentz transformations

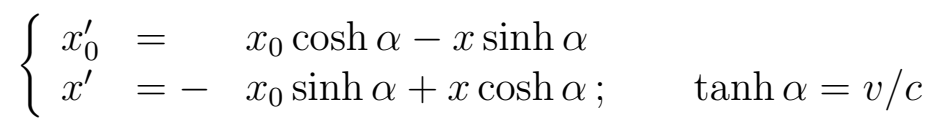

so that the new linear transformations will also form a group with a single parameter $\alpha$ and will keep invariance of the wave equation $\left(\partial^{2} / \partial x_{0}^{2}-\partial^{2} / \partial x^{2}\right) f=0$. Guided by the conformal invariance of the electrodynamic equations, we insert an additional scale transformation into (1). As a result, in place of (1), we obtain the generalized Lorentz transformations in the form

$$
\left\{\begin{array}{l}
x_{0}^{\prime}=e^{-r \alpha} \quad\left(\quad x_{0} \cosh \alpha-x \sinh \alpha\right) \\
x^{\prime}=e^{-r \alpha} \quad\left(-x_{0} \sinh \alpha+x \cosh \alpha\right),
\end{array}\right.
$$

where $r$ is a dimensionless parameter of the scale transformation. Since according to (2) the relation of the group parameter $\alpha$ to the velocity $v$ of the primed frame remains the same, i.e. $\tanh \alpha=v / c,(2)$ can be rewritten as follows

$$
\left\{\begin{aligned}
x_{0}^{\prime} & =\left(\frac{1-v / c}{1+v / c}\right)^{r / 2} \frac{x_{0}-(v / c) x}{\sqrt{1-v^{2} / c^{2}}} \\
x^{\prime} & =\left(\frac{1-v / c}{1+v / c}\right)^{r / 2} \frac{x-(v / c) x_{0}}{\sqrt{1-v^{2} / c^{2}}} .
\end{aligned}\right.
$$


Obviously, in contrast to (1), the "generalized Lorentz transformations" (2) or (3) do not leave invariant the pseudo-Euclidean metric $d s^{2}=d x_{0}^{2}-d x^{2}$ but conformally modify it. Therefore, the question arises as to what the metric of an event space invariant under such "generalized Lorentz transformations" is. The rigorous solution to this problem is

$$
d s^{2}=\left[\frac{\left(d x_{0}-d x\right)^{2}}{d x_{0}^{2}-d x^{2}}\right]^{r}\left(d x_{0}^{2}-d x^{2}\right)
$$

Not being a quadratic form but a homogeneous function of the coordinate differentials of degree two, the metric (4) falls into the category of Finsler metrics. It describes a flat but anisotropic event space. 9 . As long as we deal with 2D anisotropic space, its anisotropy manifests itself in the noninvariance of the metric (4) under the reflections $x_{0} \rightarrow-x_{0}$ or $x \rightarrow-x$. If $r=0$, then the anisotropy disappears. In this case, the event space becomes isotropic while the "generalized Lorentz transformations" (3) reduce to the usual Lorentz transformations. However, if $r \neq 0$ characterizing the magnitude of space anisotropy, is sufficiently small, then the additional dilatation of space-time, which distinguishes the generalized Lorentz transformations from the usual ones, becomes markedly different from unity only at relative velocities of the inertial frames extremely close to the velocity of light. In the physics of ultra-high energy cosmic rays we deal with precisely such a situation. Therefore, the use of the "generalized Lorentz transformations" instead of the usual ones makes it possible, in principle, to remove the discrepancy between theory and experiment in this field; this may be regarded as a hint towards a local anisotropy of space.

According to (4), the parameter $r$ is limited by the condition $|r|<1$. In this case, due to equation $d s^{2}=0$, the velocity of light is the same in both directions of the $x$ - axis and is equal to $c$ in spite of the presence of a space-time anisotropy. Both relations (3) incorporate the same power-type scale factor canceling out when the second relation (3) is divided by the first one. As a result the Einstein formula, which describes the addition of $3 \mathrm{D}$ velocities, is reproduced:

$$
V=\frac{V^{\prime}+v}{1+V^{\prime} v / c^{2}}
$$

where $v$ is the velocity of the primed frame.

Certainly, the 2D model (4) of a flat anisotropic event space is of methodical interest only and must be generalized to the $4 \mathrm{D}$ case. It turns out that two independent ways for such an extension exist. The first path leads to a flat anisotropic space-time with partially broken symmetry with respect to $3 \mathrm{D}$ rotations. The second way ends in a flat anisotropic space-time with entirely broken rotational symmetry. Both models are sufficiently interesting from a physical aspect.

\subsection{Partially broken isotropy}

\footnotetext{
${ }^{5}$ In accordance with the Busemann approach to the mathematical theory of Finsler spaces, a flat anisotropic space is understood as a linear normalized vector space in which the norm of a vector is determined not exclusively by its Euclidean length but also by its orientation with respect to some preferred direction.
} 
We first will discuss the model of space-time with a partially broken isotropy. The corresponding 4D Finslerian metric can be found if we note that the 2D metric (4) is a definite function of two quantities: $\left(d x_{0}^{2}-d x^{2}\right)$ and $\left(d x_{0}-d x\right)$. The first quantity is the pseudo-Euclidean square of a vector having components $\left(d x_{0}, d x\right)$. The second quantity is the pseudo-Euclidean scalar product of such a vector with a vector $\nu^{i}=(1,1)$. Replacing now the cited vectors by their $4 \mathrm{D}$ analogs with the components $\left(d x_{0}, d \boldsymbol{x}\right)$ and $\nu^{i}=(1, \boldsymbol{\nu})$, where $\boldsymbol{\nu}^{2}=1$, we arrive at

$$
\left(d x_{0}^{2}-d x^{2}\right) \rightarrow\left(d x_{0}^{2}-d \boldsymbol{x}^{2}\right) ; \quad\left(d x_{0}-d x\right) \rightarrow\left(d x_{0}-\boldsymbol{\nu} d \boldsymbol{x}\right),
$$

by means of which the $4 \mathrm{D}$ metric is obtained from the $2 \mathrm{D}$ metric (4)

$$
d s^{2}=\left[\frac{\left(d x_{0}-\boldsymbol{\nu} d \boldsymbol{x}\right)^{2}}{d x_{0}^{2}-d \boldsymbol{x}^{2}}\right]^{r}\left(d x_{0}^{2}-d \boldsymbol{x}^{2}\right) .
$$

This Finslerian metric depends on two constant parameters $r$ and $\boldsymbol{\nu}$ and describes a flat anisotropic space-time with partially broken rotational symmetry. Instead of the 3-parameter group of rotations of Minkowski space, the space-time (6) admits only the 1-parameter group of rotations about the unit vector $\boldsymbol{\nu}$, which indicates a preferred direction in 3D space. No changes occur for translational symmetry: space-time translations leave the metric (6) invariant. As regards the transformations linking the various inertial frames, the usual Lorentz boosts modify the metric (6). Therefore, they do not belong to the isometry group of the space-time (6). By proper use of them, however, invariance transformations for the metric (6) can be constructed. The corresponding transformations, named "generalized Lorentz transformations", will be the following

$$
x^{i}=D(\boldsymbol{v}, \boldsymbol{\nu}) R_{j}^{i}(\boldsymbol{v}, \boldsymbol{\nu}) L_{k}^{j}(\boldsymbol{v}) x^{k} .
$$

In order to demonstrate how the invariance of the metric (6) is realized under the transformations (7), we first carry out the transformation using the matrix $L_{k}^{j}(\boldsymbol{v})$, which represents a usual Lorentz boost (the given matrix naturally depends on the velocity $\boldsymbol{v}$ of a moving frame). As a result, in expression (6) only the Minkowskian scalar product $d x^{0}-\boldsymbol{\nu} d \boldsymbol{x}$ will change its form since the components $(1, \boldsymbol{\nu})$ of the 4 -vector $\nu^{i}$ will change. The vector $\nu^{i}$ was initially defined as light-like $\left(1-\boldsymbol{\nu}^{2}=0\right)$. It will remain the same after the boost, i.e. it will remain light-like although both the time and spatial components are changed by a scale factor. In addition, the spatial component of the 4 -vector $\nu^{i}$ will change its direction due to the rotation about the vector $[\boldsymbol{v} \boldsymbol{\nu}]$ through an angle of relativistic aberration

$$
\varphi=\arccos \left\{1-\frac{\left(1-\sqrt{1-\boldsymbol{v}^{2} / c^{2}}\right)[\boldsymbol{v} \boldsymbol{\nu}]^{2}}{(1-\boldsymbol{v} \boldsymbol{\nu} / c) \boldsymbol{v}^{2}}\right\}
$$

Therefore, having carried out (according to (7)), subsequent to the Lorentz boost, an additional rotation $R_{j}^{i}(\boldsymbol{v}, \boldsymbol{\nu})$ of the space axes of the moving frame through the angle (8) about the vector $[\boldsymbol{v} \boldsymbol{\nu}]$, we regain for the spatial component of the 4 -vector $\nu^{i}$ its initial orientation with respect to the space axes. The net result of the transformations 
performed will be the fact that the form $\left(d x_{0}^{2}-d \boldsymbol{x}^{2}\right)$ will not be changed while the form $\left(d x_{0}-\boldsymbol{\nu} d \boldsymbol{x}\right)$ will be altered minimally: its new representation will be distinguished from the initial one only by a scale factor dependent on $\boldsymbol{v}$ and $\boldsymbol{\nu}$. If now, as prescribed by (7), we perform also the dilatation transformation of the event coordinates using the matrix

$$
D(\boldsymbol{v}, \boldsymbol{\nu})=\left(\frac{1-\boldsymbol{v} \boldsymbol{\nu} / c}{\sqrt{1-\boldsymbol{v}^{2} / c^{2}}}\right)^{r} I
$$

where $I$ is the unit matrix, then within the square brackets in (6) no additional scale factor will appear ?, while the scale factor, which occurred there at the previous stage of transformations, will be completely cancelled out by a scale factor arising from the dilatation transformation of the expression $\left(d x_{0}^{2}-d \boldsymbol{x}^{2}\right)$. The result is that the generalized Lorentz transformations determined by (7) do indeed leave the metric (6) invariant.

In contrast to Lorentz boosts, the generalized transformations (7) make up a 3parameter noncompact group with generators $X_{1}, X_{2}, X_{3}$. Thus, with the inclusion of the 1-parameter group of rotations about the preferred direction $\boldsymbol{\nu}$ and of the 4-parameter group of translations, the inhomogeneous group of isometries of the space (6) turns out to have 8-parameters. In order to obtain the simplest representation for its generators, it is sufficient to choose a third space axis along $\boldsymbol{\nu}$ and then to make use of the infinitesimal form of the transformations (7). As a result,

$$
\begin{array}{ll}
X_{1}=-\left(x^{1} p_{0}+x^{0} p_{1}\right)-\left(x^{1} p_{3}-x^{3} p_{1}\right), & \\
X_{2}=-\left(x^{2} p_{0}+x^{0} p_{2}\right)+\left(x^{3} p_{2}-x^{2} p_{3}\right), & \\
X_{3}=-r x^{i} p_{i}-\left(x^{3} p_{0}+x^{0} p_{3}\right), & p_{i}=\partial / \partial x^{i} . \\
R_{3}=x^{2} p_{1}-x^{1} p_{2} ; &
\end{array}
$$

The generators (10) satisfy the commutation relations

$$
\begin{array}{llll}
{\left[X_{1} X_{2}\right]=0,} & {\left[R_{3} X_{3}\right]=0,} & & \\
{\left[X_{3} X_{1}\right]=X_{1},} & {\left[R_{3} X_{1}\right]=X_{2},} & & \\
{\left[X_{3} X_{2}\right]=X_{2},} & {\left[R_{3} X_{2}\right]=-X_{1} ;} & & \\
{\left[p_{i} p_{j}\right]=0 ;} & & & \\
{\left[X_{1} p_{0}\right]=p_{1},} & {\left[X_{2} p_{0}\right]=p_{2},} & {\left[X_{3} p_{0}\right]=r p_{0}+p_{3},} & {\left[R_{3} p_{0}\right]=0,} \\
{\left[X_{1} p_{1}\right]=p_{0}+p_{3},} & {\left[X_{2} p_{1}\right]=0,} & {\left[X_{3} p_{1}\right]=r p_{1},} & {\left[R_{3} p_{1}\right]=p_{2},} \\
{\left[X_{1} p_{2}\right]=0,} & {\left[X_{2} p_{2}\right]=p_{0}+p_{3},} & {\left[X_{3} p_{2}\right]=r p_{2},} & {\left[R_{3} p_{2}\right]=-p_{1},} \\
{\left[X_{1} p_{3}\right]=-p_{1},} & {\left[X_{2} p_{3}\right]=-p_{2},} & {\left[X_{3} p_{3}\right]=r p_{3}+p_{0},} & {\left[R_{3} p_{3}\right]=0 .}
\end{array}
$$

From (11), we conclude in particular that the homogeneous isometry group of the space (6) contains 4 parameters (the generators $X_{1}, X_{2}, X_{3}, R_{3}$ ). Being a subgroup of the conformal group, it is isomorphic to the corresponding 4-parameter subgroup of the homogeneous Lorentz group (with the generators $X_{1}, X_{2},\left.X_{3}\right|_{r=0}, R_{3}$ ). Since the 6-parameter homogeneous Lorentz group has no 5-parameter subgroup [21] while the 4-parameter subgroup is unique (up to isomorphisms), the transition from Minkowski space to the event

\footnotetext{
${ }^{6}$ since the ratio, enclosed within these brackets, is homogeneous of degree zero with respect to the coordinate differentials
} 
space (6) implies a minimum of symmetry-breaking of the Lorentz symmetry. Some types of Finslerian spaces with more radical breaking of the Lorentz symmetry are considered in $[22-24]$.

A remarkable property of the anisotropic event space (6) is the fact that it keeps the conformal structure (light cones) of Minkowski space, i.e. light propagates according to the equation $d x_{0}^{2}-d \boldsymbol{x}^{2}=0$. Therefore, the velocity of light is independent of the direction of its propagation and is equal to $c$. It thus appears that the square of the distance $d l^{2}$ between adjacent points of 3D space, determined by means of exchange of light signals], is expressed by the formula $d l^{2}=d \boldsymbol{x}^{2}$. Thus, although in the 3D space there is a preferred direction $\boldsymbol{\nu}$, its geometry remains Euclidean. But, what does the anisotropy physically manifest itself in? First of all, it affects the dependence of proper time of a moving clock by including the direction of its velocity in addition to the magnitude. According to (6), the interval $d \tau$ of proper time read by the clock moving with a velocity $\boldsymbol{v}$, is related to the time interval $d t$ read by a clock at rest by the relation $d \tau=(d \tau / d t) d t$, where

$$
\frac{d \tau}{d t}=\left(\frac{1-\boldsymbol{v} \boldsymbol{\nu} / c}{\sqrt{1-\boldsymbol{v}^{2} / c^{2}}}\right)^{r} \sqrt{1-\boldsymbol{v}^{2} / c^{2}}
$$

It can be seen from Fig. 1, that, in contrast to Minkowski space (for which: $r=0$, $\left.(d \tau / d t)\right|_{r=0}=\sqrt{1-v^{2} / c^{2}} \leq 1$ and, hence, the moving clock is always slow in comparison with the clock at rest), in the anisotropic space the time dilatation factor $\left.(d \tau / d t)\right|_{r>0}$ can take on values greater than unity. Therefore, at some of its velocities the clock moving in the anisotropic space is fast in comparison with the clock at rest. However, having returned to its starting point, it will necessarily run behind the clock at rest. Consequently, at $r>0$ inertial motion is still uniform and along a straight line.

Along with the time dilatation factor the anisotropy of space also affects the Doppler shift. In place of the usual relativistic formula, now the modified relation [25] holds:

$$
\omega=\omega^{\prime} \frac{\sqrt{1-\boldsymbol{v}^{2} / c^{2}}}{1-\boldsymbol{v} \boldsymbol{e} / c}\left(\frac{1-\boldsymbol{v} \boldsymbol{\nu} / c}{\sqrt{1-\boldsymbol{v}^{2} / c^{2}}}\right)^{r},
$$

where $r$ is the magnitude of space anisotropy, $\boldsymbol{v}$ the velocity of a moving frame, $\omega^{\prime}$ the frequency of a ray with respect to it, and $\omega, \boldsymbol{e}$ and $\boldsymbol{\nu}$ are the frequency, direction of the ray and the preferred direction in an initial frame.

Precision measurements of the Doppler effect by use of the Mössbauer effect were suggested and have since been made (Cf. [26-28]). Of special interest here is the experiment of ref. [27] which consisted in measuring a relative frequency shift $\Delta \omega / \omega=\left(\omega_{a}-\omega_{s}\right) / \omega_{s}$ between a Mössbauer source and an absorber placed at equal and diametrically opposite distances from the center of a rapidly rotating rotor. For the quantity $\Delta \omega / \omega$, the prerelativistic theory of absolute aether (PR), SR and the relativistic theory of locally

\footnotetext{
${ }^{7}$ As regards the additional tachyon solution $d x_{0}-\boldsymbol{\nu} d \boldsymbol{x}=0$ of the equation $d s^{2}=0$, it does not admit the reflection operation $d \boldsymbol{x} \rightarrow-d \boldsymbol{x}$. Therefore, there is no algorithm for determining the 3D distances based on exchange of tachyon signals.
} 
anisotropic space (AR), respectively, give the following predictions to within $v^{2} / c^{2}$

$$
\begin{aligned}
& (\Delta \omega / \omega)^{P R}=2 \boldsymbol{w} \boldsymbol{v}_{a} / c^{2}, \\
& (\Delta \omega / \omega)^{S R}=0, \\
& (\Delta \omega / \omega)^{A R}=2 \boldsymbol{r} c \boldsymbol{\nu} \boldsymbol{v}_{a} / c^{2},
\end{aligned}
$$

where $\boldsymbol{w}$ is the velocity of the aether wind and $\boldsymbol{v}_{a}$ the velocity of the absorber. Comparing (14) and (16), we may regard the quantity $r c \boldsymbol{\nu}$ in a sense as the velocity of the aether wind. It must be noted, however, that $r c \boldsymbol{\nu}$ is an invariant of the generalized Lorentz transformations. In the experiment of ref. [27], no aether wind was found. As a result, an upper limit, specified in ref. [29], was obtained for the velocity of the aether wind. In terms of the relativistic theory of anisotropic space-time this constraint signifies that the value of anisotropy $r<5 \times 10^{-10}$. At the present time, due to the use of radically new rotors developed at the I.T.E.P. (Moscow) and of Mössbauer sources with a much narrower line width, it is possible to lower the minimally detectable value of anisotropy at least by three orders of magnitude. Therefore, a repetition of the experiment [27] would now be interesting.

\subsection{Modification of fundamental relativistic equations in the anisotropic space}

All fundamental relativistic equations are invariant under the transformations of the Poincaré group, the isometry group of Minkowski space. If the event space is described by the Finslerian metric (6), then the complete inhomogeneous group of its isometries turns out to be an 8-parameter group. In this a case, the fundamental relativistic equations must be modified in accordance with the requirement of invariance under this group.

The requirement just formulated represents a generalization of the special principle of relativity for the locally anisotropic space-time. Since the 8-parameter group in question is incorporated in the conformal group and the standard relativistic equations relating to massless particles are conformally invariant, then only such equations continue to work in the locally anisotropic space as well. The remaining relativistic equations, in particular, the standard equations of relativistic mechanics are neither conformally invariant nor invariant under the transformations belonging to the 8-parameter linear subgroup of the conformal group. Thus, such equations need modification. We now consider the required modification [30] for the equations of mechanics.

The equations of relativistic mechanics, which satisfy the special principle of relativity for the locally anisotropic space, can be obtained if in the action integral

$$
S=-m c \int_{a}^{b} d s
$$

we replace the Minkowskian expression for $d s$ by the Finslerian expression (6). As a result, the Lagrangian function corresponding to a free particle in the locally anisotropic space, takes the form

$$
L=-m c^{2}\left(\frac{1-\boldsymbol{v} \boldsymbol{\nu} / c}{\sqrt{1-\boldsymbol{v}^{2} / c^{2}}}\right)^{r} \sqrt{1-\boldsymbol{v}^{2} / c^{2}}
$$


This Lagrangian leads to the following expressions for the momentum $\boldsymbol{p}=\partial L / \partial \boldsymbol{v}$ and the energy $E=\boldsymbol{p} \boldsymbol{v}-L$ of a relativistic particle

$$
\begin{gathered}
E=\frac{m c^{2}}{\sqrt{1-\boldsymbol{v}^{2} / c^{2}}}\left(\frac{1-\boldsymbol{v} \boldsymbol{\nu} / c}{\sqrt{1-\boldsymbol{v}^{2} / c^{2}}}\right)^{r}\left[1-r+r \frac{1-\boldsymbol{v}^{2} / c^{2}}{1-\boldsymbol{v} \boldsymbol{\nu} / c}\right], \\
\boldsymbol{p}=\frac{m c}{\sqrt{1-\boldsymbol{v}^{2} / c^{2}}}\left(\frac{1-\boldsymbol{v} \boldsymbol{\nu} / c}{\sqrt{1-\boldsymbol{v}^{2} / c^{2}}}\right)^{r}\left[(1-r) \boldsymbol{v} / c+r \boldsymbol{\nu} \frac{1-\boldsymbol{v}^{2} / c^{2}}{1-\boldsymbol{v} \boldsymbol{\nu} / c}\right] .
\end{gathered}
$$

It can be verified by direct substitution that energy and momentum are related by the relation

$$
\left[\frac{(E / c-\boldsymbol{p} \boldsymbol{\nu})^{2}}{E^{2} / c^{2}-\boldsymbol{p}^{2}}\right]^{-r}\left(E^{2} / c^{2}-\boldsymbol{p}^{2}\right)=m^{2} c^{2}(1-r)^{(1-r)}(1+r)^{(1+r)} .
$$

This relation determines the square of the Finslerian length of the 4-momentum $p$. In passing from one inertial frame to another its components $p^{0}=E / c$ and $\boldsymbol{p}$ must transform such as to guarantee invariance of the form (21). We have shown above that the invariance of the Finslerian metric (6) is established by the generalized Lorentz transformations (7). From the comparison of (21) and (6), the invariance of (21) results from the transformations

$$
p^{i}=D^{-1} R_{j}^{i} L_{k}^{j} p^{k}
$$

where the matrices $L_{k}^{j}$ and $R_{j}^{i}$ are the same as in (7), while

$$
D^{-1}=\left(\frac{1-\boldsymbol{v} \boldsymbol{\nu} / c}{\sqrt{1-\boldsymbol{v}^{2} / c^{2}}}\right)^{-r} I .
$$

Thus, under generalized Lorentz transformations the scale transformation (23) for momenta is inverse to the corresponding scale transformation (9) for the coordinates of events. Consequently, the phase of a plane wave is an invariant of the generalized Lorentz transformations.

Eq. (19) determines the dependence of the energy $E$ of a free particle, present in the anisotropic space, on both the magnitude and the direction of its velocity $\boldsymbol{v}$. At $\boldsymbol{v}=0$ the energy reaches its absolute minimum $E_{0}=m c^{2}$. As regards the momentum $\boldsymbol{p}$, its direction, according to (20), does not coincide with the direction of the velocity of a massive particle. Even in the case $\boldsymbol{v}=0$, the momentum of a particle does not vanish; there remains a "rest momentum" $\boldsymbol{p}_{0}=r m c \boldsymbol{\nu}$. Massless particles have no such property; for them, as in SR, $v=c$ and $E^{2} / c^{2}-\boldsymbol{p}^{2}=0$.

In the space of 4 -momenta $p$ the relation (21) is the equation of mass shell. It appears as a deformed two-sheeted hyperboloid inscribed into a cone $p^{02}-\boldsymbol{p}^{2}=0$. For the upper sheet of such a "hyperboloid" $p^{0}$ reaches its absolute minimum $p_{\min }^{0}=E_{0} / c=m c$ at $\boldsymbol{p}=\boldsymbol{p}_{0}=r m c \boldsymbol{\nu}$. For the lower sheet, $p^{0}$ reaches its absolute maximum $p_{\max }^{0}=-m c$ at $\boldsymbol{p}=-r m c \boldsymbol{\nu}$. In order to display the mass shell graphically, let us introduce the dynamic 4 -velocity $u=p / m c$ in place of $p$. We also put $c=1$ and choose the coordinate axes such that $\boldsymbol{\nu}=(1,0,0)$. If we confine our consideration to the case of two-dimensional motion 
and use polar coordinates: $0 \leq v<1 ; 0 \leq \alpha<2 \pi$, in which $\boldsymbol{v}=(v \cos \alpha, v \sin \alpha, 0)$, then, according to (21), (20), (19), the equation of (the positive frequency part of) the mass shell

$$
\left[\frac{\left(u^{0}-u^{1}\right)^{2}}{\left(u^{0}\right)^{2}-\left(u^{1}\right)^{2}-\left(u^{2}\right)^{2}}\right]^{-r}\left[\left(u^{0}\right)^{2}-\left(u^{1}\right)^{2}-\left(u^{2}\right)^{2}\right]=(1-r)^{(1-r)}(1+r)^{(1+r)}
$$

can be written in the following parametric form (parameters $v, \alpha$ )

$$
\begin{aligned}
& u^{1}=\left(\frac{1-v \cos \alpha}{\sqrt{1-v^{2}}}\right)^{r}\left[(1-r) v \cos \alpha+\frac{r\left(1-v^{2}\right)}{1-v \cos \alpha}\right] / \sqrt{1-v^{2}} \\
& u^{2}=\left(\frac{1-v \cos \alpha}{\sqrt{1-v^{2}}}\right)^{r} \frac{(1-r) v \sin \alpha}{\sqrt{1-v^{2}}} \\
& u^{0}=\left(\frac{1-v \cos \alpha}{\sqrt{1-v^{2}}}\right)^{r}\left[1-r+\frac{r\left(1-v^{2}\right)}{1-v \cos \alpha}\right] / \sqrt{1-v^{2}} .
\end{aligned}
$$

The results of calculations using (25) are presented in Fig. 2. As, according to (24), the mass shell is symmetric with respect to the plane $u_{2}=0$, the range of variation of the angle $\alpha$ was limited in Fig. 2 by the condition $0 \leq \alpha \leq \pi$.

Being an intrinsic property of space, anisotropy is independent of the magnitude of relative velocities. Therefore, also nonrelativistic mechanics as a whole is different from the Newtonian case. In fact, in the nonrelativistic limit the following expressions are obtained from (19) and (20)

$$
\begin{aligned}
& E=m c^{2}+(1-r) \frac{m \boldsymbol{v}^{2}}{2}+r(1-r) \frac{m(\boldsymbol{v} \boldsymbol{\nu})^{2}}{2}, \\
& \boldsymbol{p}=r m c \boldsymbol{\nu}+(1-r) m \boldsymbol{v}+r(1-r) m(\boldsymbol{v} \boldsymbol{\nu}) \boldsymbol{\nu} .
\end{aligned}
$$

Since within the framework of nonrelativistic mechanics the rest mass $m$ is an additive quantity, the occurrence of the constant terms $m c^{2}$ and $r m c \boldsymbol{\nu}$ in (26) and (27) does not affect the conservation laws and the equations of motion. As a result, these terms can be omitted, and the kinetic energy and kinetic momentum, read off from (26) and (27), are

$$
T=\frac{1}{2} \mathfrak{M}_{\alpha \beta} v^{\alpha} v^{\beta}, \quad p_{\alpha}=\mathfrak{M}_{\alpha \beta} v^{\beta}
$$

where

$$
\mathfrak{M}_{\alpha \beta}=m(1-r)\left(\delta_{\alpha \beta}+r \nu_{\alpha} \nu_{\beta}\right) \text {. }
$$

Differentiating the second relation in (28) with respect to time, and using as definition of force the derivative of momentum, we find that Newton's second law in anisotropic space has the form

$$
\mathfrak{M}_{\alpha \beta} a^{\beta}=F_{\alpha}
$$

$(\alpha=1,2,3)$. Thus the inertial properties of a nonrelativistic particle in anisotropic space is specified by a tensor of inertial mass (29); its motion is analogous to the motion of 
a quasiparticle in a crystalline medium. Equations as (30) were also discussed in the framework of nonrelativistic Machian theories ([31]).

Irrespective of which closed physical system is considered - nonrelativistic or relativistic - , according to Noether's first theorem [32], there exist eight additive integrals of motion corresponding to the eight independent symmetry transformations of the space-time (6). Conservation of the total 4-momentum follows from the 4-parameter translational symmetry of (6); conservation of three more quantities determining the coordinates of the center of inertia corresponds to the 3-parameter generalized Lorentz symmetry of (6); and, finally, conservation of the projection of the total angular momentum of the system onto the preferred direction $\boldsymbol{\nu}$ corresponds to the symmetry of (6) with respect to rotations about $\boldsymbol{\nu}$.

The conservation law of total momentum manifests itself differently in isotropic and anisotropic spaces. As an elementary example, consider the elastic collision of two particles in isotropic space, one of which at first was at rest. The conservation law of total momentum then makes the tracks of the particle coplanar. For the same process but now in anisotropic space, where the directions of velocities and, hence, of the tracks of particles do not coincide with the directions of their momenta, the conservation law of total momentum does not lead to the fact that all the three tracks must necessarily lie in the same plane. However, since the amount of the deviation from coplanarity is a function of the magnitude of space anisotropy, possible effects of noncoplanarity should be searched for in regions where the magnitude of local anisotropy is significantly greater than its mean value ( i.e. greater than $10^{-10}$ ). Such a situation may obtain in the vicinity of very large masses, for example, near the Sun. It seems reasonable to test this assumption with a corresponding detector on a space vehicle able to identify elementary events with nonstandard kinematics.

Having mentioned that the magnitude of local anisotropy near massive bodies is greater than that farther away, we thus strayed from the model of space whose anisotropy is constant - always and everywhere. Introducing a field of anisotropy against the background of space-time curved by matter would be a further step in the construction of a theory of locally anisotropic space-time. We will turn to this problem in Section 6. In the next Section, we consider another type of a possible breaking of local isotropy, viz. a complete loss of symmetry with respect to the group of $3 \mathrm{D}$ rotations. 


\section{FINSLERIAN METRIC OF A FLAT SPACE-TIME WITH ENTIRELY BROKEN 3D ISOTROPY}

It was demonstrated in Section 2 that the homogeneous isometry group of a flat spacetime with a partially broken local isotropy, i.e. the homogeneous isometry group of the space (6), is a 4-parameter group and includes, apart from 3-parameter generalized Lorentz transformations (7), the 1-parameter group of rotations about the preferred direction $\boldsymbol{\nu}$. We now try to construct a geometric model of a flat space-time, the homogeneous isometry group of which would only consist of noncompact 3-parameter transformations of relativistic symmetry. For the solution of this problem, the following observation is important.

Consider the metric (6) in the limiting case $r=1$. In this case

$$
d s=d x_{0}-\boldsymbol{\nu} d \boldsymbol{x}
$$

and since $\boldsymbol{\nu}=$ const, $d s$ turns out to be a total differential; the action (17) for a free particle of mass $m$ is no longer dependent on the shape of the world line connecting the points $a$ and $b$. All this means that at $r=1$ a massive particle loses its inertia. This can be illustrated by Eq. (29) which determines the inertial mass tensor $\mathfrak{M}_{\alpha \beta}$, and also by Eqs. (19) and (20) which determine the dependence of the energy $E$ and the momentum $\boldsymbol{p}$ on the particle velocity $\boldsymbol{v}$. From these formulae, at $r=1$, it follows that $\mathfrak{M}_{\alpha \beta}=0$ while $E$ and $\boldsymbol{p}$ become no longer dependent on $\boldsymbol{v}$ and become equal to the corresponding constants $m c^{2}$ and $m c \boldsymbol{\nu}$. At $r=1$, apart from inertness, the notion of spatial extension disappears, which is due to the absence of a light cone and, hence, of the possibility itself for determining spatial distances with the aid of exchange of light signals. As a result, in the space-time (31) there remains a single physical characteristic - time duration $d s$ and it should be regarded as an interval of absolute time.

Since the "metric" (31) is a special case of the metric (6), all transformations leaving invariant the metric (6) leave invariant the "metric" (31) as well. This likewise applies to the transformations (7), if in them $r=1$ is set. It can readily be seen, however, that in comparison with (6) the "metric" (31) possesses an additional symmetry. Its existence becomes evident after substitution of the variables

$$
\nu_{1} x_{1} \rightarrow x_{1}, \quad \nu_{2} x_{2} \rightarrow x_{2}, \quad \nu_{3} x_{3} \rightarrow x_{3} ; \quad \nu_{1}, \nu_{2}, \nu_{3} \neq 0
$$

as a result of which Eq. (31) takes the form

$$
d s=d x_{0}-d x_{1}-d x_{2}-d x_{3} .
$$

Thus our observation (suggested by invariance of the expression (4) under (2) at $r=1$ ) consists in the fact that the additional symmetry of the 1-form (32) is realized as its invariance under the following three independent 1-parameter groups of transformations

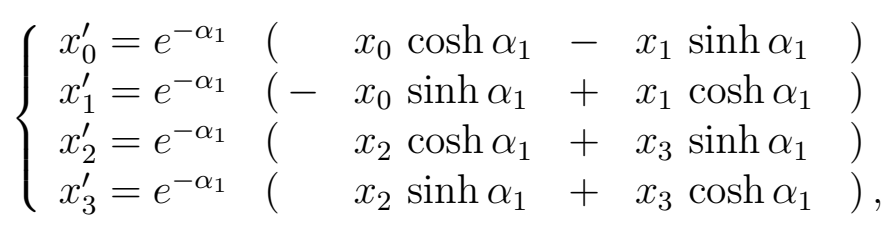




$$
\begin{aligned}
& \left\{\begin{array}{l}
x_{0}^{\prime}=e^{-\alpha_{2}} \quad\left(\quad x_{0} \cosh \alpha_{2}-x_{2} \sinh \alpha_{2}\right) \\
x_{1}^{\prime}=e^{-\alpha_{2}} \quad\left(\begin{array}{l}
x_{1} \cosh \alpha_{2}+x_{3} \sinh \alpha_{2}
\end{array}\right) \\
x_{2}^{\prime}=e^{-\alpha_{2}}\left(-x_{0} \sinh \alpha_{2}+x_{2} \cosh \alpha_{2}\right) \\
x_{3}^{\prime}=e^{-\alpha_{2}} \quad\left(\begin{array}{l}
x_{1} \sinh \alpha_{2}+x_{3} \cosh \alpha_{2}
\end{array}\right),
\end{array}\right. \\
& \left\{\begin{array}{l}
x_{0}^{\prime}=e^{-\alpha_{3}} \quad\left(\begin{array}{l}
x_{0} \cosh \alpha_{3}-x_{3} \sinh \alpha_{3}
\end{array}\right) \\
x_{1}^{\prime}=e^{-\alpha_{3}} \quad\left(\begin{array}{l}
x_{1} \cosh \alpha_{3}+x_{2} \sinh \alpha_{3}
\end{array}\right) \\
x_{2}^{\prime}=e^{-\alpha_{3}} \quad\left(\begin{array}{l}
x_{1} \sinh \alpha_{3}+x_{2} \cosh \alpha_{3}
\end{array}\right) \\
x_{3}^{\prime}=e^{-\alpha_{3}} \quad\left(-x_{0} \sinh \alpha_{3}+x_{3} \cosh \alpha_{3}\right) .
\end{array}\right.
\end{aligned}
$$

Note that each of the groups (33)-(35) is represented by nonorthogonal transformations. For example, the transformations (33) change the angle between the axes $x_{2}$ and $x_{3}$. Apart from the 1-form (32), the transformations (33), (34) and (35), respectively leave invariant the 1 -forms

$$
\begin{gathered}
d s=d x_{0}-d x_{1}+d x_{2}+d x_{3} \\
d s=d x_{0}+d x_{1}-d x_{2}+d x_{3}
\end{gathered}
$$

and

$$
d s=d x_{0}+d x_{1}+d x_{2}-d x_{3} .
$$

Let us make now the following step by introducing into (33)-(35) a dependence of the dilatations $e^{-\alpha_{1}}, e^{-\alpha_{2}}$, and $e^{-\alpha_{3}}$ on three independent parameters $r_{1}, r_{2}$ and $r_{3}$, respectively. As a result of such generalization we arrive at three independent 1-parameter groups of transformations, corresponding to eqs. (33)-(35) but in which the factors $e^{-\alpha_{i}}$ are replaced by $e^{-r_{i} \cdot \alpha_{i}}$, respectively ( $\alpha_{i}$ still act as group parameters). These transformations act on the 1-forms (32), (36-38) in the following way

$$
\begin{aligned}
& \left\{\begin{array}{rl}
d x_{0}^{\prime}-d x_{1}^{\prime}-d x_{2}^{\prime}-d x_{3}^{\prime}=e^{\left(1-r_{1}\right) \alpha_{1}} & \left(d x_{0}-d x_{1}-d x_{2}-d x_{3}\right) \\
d x_{0}^{\prime}-d x_{1}^{\prime}+d x_{2}^{\prime}+d x_{3}^{\prime}=e^{\left(1-r_{1}\right) \alpha_{1}} & \left(d x_{0}-d x_{1}+d x_{2}+d x_{3}\right) \\
d x_{0}^{\prime}+d x_{1}^{\prime}-d x_{2}^{\prime}+d x_{3}^{\prime}=e^{-\left(1+r_{1}\right) \alpha_{1}} & \left(d x_{0}+d x_{1}-d x_{2}+d x_{3}\right) \\
d x_{0}^{\prime}+d x_{1}^{\prime}+d x_{2}^{\prime}-d x_{3}^{\prime}=e^{-\left(1+r_{1}\right) \alpha_{1}} & \left(d x_{0}+d x_{1}+d x_{2}-d x_{3}\right)
\end{array},\right. \\
& \left\{\begin{array}{rl}
d x_{0}^{\prime}-d x_{1}^{\prime}-d x_{2}^{\prime}-d x_{3}^{\prime}=e^{\left(1-r_{2}\right) \alpha_{2}} & \left(d x_{0}-d x_{1}-d x_{2}-d x_{3}\right) \\
d x_{0}^{\prime}-d x_{1}^{\prime}+d x_{2}^{\prime}+d x_{3}^{\prime}=e^{-\left(1+r_{2}\right) \alpha_{2}} & \left(d x_{0}-d x_{1}+d x_{2}+d x_{3}\right) \\
d x_{0}^{\prime}+d x_{1}^{\prime}-d x_{2}^{\prime}+d x_{3}^{\prime}=e^{\left(1-r_{2}\right) \alpha_{2}} & \left(d x_{0}+d x_{1}-d x_{2}+d x_{3}\right) \\
d x_{0}^{\prime}+d x_{1}^{\prime}+d x_{2}^{\prime}-d x_{3}^{\prime}=e^{-\left(1+r_{2}\right) \alpha_{2}} & \left(d x_{0}+d x_{1}+d x_{2}-d x_{3}\right)
\end{array},\right. \\
& \left\{\begin{aligned}
d x_{0}^{\prime}-d x_{1}^{\prime}-d x_{2}^{\prime}-d x_{3}^{\prime}=e^{\left(1-r_{3}\right) \alpha_{3}} & \left(d x_{0}-d x_{1}-d x_{2}-d x_{3}\right) \\
d x_{0}^{\prime}-d x_{1}^{\prime}+d x_{2}^{\prime}+d x_{3}^{\prime}=e^{-\left(1+r_{3}\right) \alpha_{3}} & \left(d x_{0}-d x_{1}+d x_{2}+d x_{3}\right) \\
d x_{0}^{\prime}+d x_{1}^{\prime}-d x_{2}^{\prime}+d x_{3}^{\prime}=e^{-\left(1+r_{3}\right) \alpha_{3}} & \left(d x_{0}+d x_{1}-d x_{2}+d x_{3}\right) \\
d x_{0}^{\prime}+d x_{1}^{\prime}+d x_{2}^{\prime}-d x_{3}^{\prime}=e^{\left(1-r_{3}\right) \alpha_{3}} & \left(d x_{0}+d x_{1}+d x_{2}-d x_{3}\right)
\end{aligned}\right.
\end{aligned}
$$

Since, according to (39-41), there occur only scale transformations of the four introduced 1-forms, we try to seek the metric for the flat Finslerian space-time (with an entirely broken symmetry with respect to $3 \mathrm{D}$ rotations) in the form

$$
\begin{aligned}
d s & =\left(d x_{0}-d x_{1}-d x_{2}-d x_{3}\right)^{a}\left(d x_{0}-d x_{1}+d x_{2}+d x_{3}\right)^{b} \\
& \times\left(d x_{0}+d x_{1}-d x_{2}+d x_{3}\right)^{c}\left(d x_{0}+d x_{1}+d x_{2}-d x_{3}\right)^{d}
\end{aligned}
$$


where $a, b, c, d$ are some constants for the determination of which the following four conditions must be fulfilled: (i) the metric (42) should be a homogeneous function of the coordinate differentials of the first degree of homogeneity; and (ii)-(iv) the metric (42) should remain invariant under the transformations belonging to any of the three independent groups (39-41). These conditions lead to a system of four equations

$$
\left\{\begin{array}{cccccccc} 
& a+ & b & c & d=1 \\
\left(1-r_{1}\right) & a+\left(1-r_{1}\right) & b & -\left(1+r_{1}\right) & c-\left(1+r_{1}\right) & d=0 \\
\left(1-r_{2}\right) & a-\left(1+r_{2}\right) & b & -\left(1-r_{2}\right) & c-\left(1+r_{2}\right) & d=0 \\
\left(1-r_{3}\right) & a-\left(1+r_{3}\right) & b & b-\left(1+r_{3}\right) & c+\left(1-r_{3}\right) & d=0
\end{array} .\right.
$$

The determinant of the given system is equal to -16 while its solution is of the form

$$
\begin{aligned}
& a=\left(1+r_{1}+r_{2}+r_{3}\right) / 4, \quad b=\left(1+r_{1}-r_{2}-r_{3}\right) / 4, \\
& c=\left(1-r_{1}+r_{2}-r_{3}\right) / 4, \quad d=\left(1-r_{1}-r_{2}+r_{3}\right) / 4 .
\end{aligned}
$$

Thus, taking into account (42), we obtain the required expression [19] for the metric of the flat locally anisotropic space-time with entirely broken rotational symmetry.?

$$
\begin{aligned}
d s & =\left(d x_{0}-d x_{1}-d x_{2}-d x_{3}\right)^{\left(1+r_{1}+r_{2}+r_{3}\right) / 4} \\
& \times\left(d x_{0}-d x_{1}+d x_{2}+d x_{3}\right)^{\left(1+r_{1}-r_{2}-r_{3}\right) / 4} \\
& \times\left(d x_{0}+d x_{1}-d x_{2}+d x_{3}\right)^{\left(1-r_{1}+r_{2}-r_{3}\right) / 4} \\
& \times\left(d x_{0}+d x_{1}+d x_{2}-d x_{3}\right)^{\left(1-r_{1}-r_{2}+r_{3}\right) / 4}
\end{aligned}
$$

The anisotropy of the Finslerian space (43) is now specified by even the three parameters $r_{1}, r_{2}, r_{3}$ which satisfy the conditions

$$
\begin{array}{ll}
1+r_{1}+r_{2}+r_{3}>0, & 1+r_{1}-r_{2}-r_{3}>0 \\
1-r_{1}+r_{2}-r_{3}>0, & 1-r_{1}-r_{2}+r_{3}>0
\end{array}
$$

These conditions ensure the fact that the section of a light cone by hyperplane $d x_{0}=$ const is a closed convex surface. This, in turn, ensures the applicability of the procedure of exchange of light signals for determining 3D distances.

According to (44), the permissible values of the parameters $r_{1}, r_{2}, r_{3}$ fill the inner region of a regular tetrahedron with the vertices at the points

$$
\begin{array}{ll}
\left(r_{1}=1, r_{2}=1, r_{3}=1\right) ; & \left(r_{1}=1, r_{2}=-1, r_{3}=-1\right) ; \\
\left(r_{1}=-1, r_{2}=1, r_{3}=-1\right) ; & \left(r_{1}=-1, r_{2}=-1, r_{3}=1\right) .
\end{array}
$$

At these four points the metric (43) degenerates into the corresponding 1-forms (32), (3638 ), i.e. into the total differentials of absolute time. We now recall that the metric (6) of the flat locally anisotropic space-time with the partially broken 3D isotropy also degenerates, at $r=1$, into the total differential of absolute time. This suggests that absolute time is not

\footnotetext{
${ }^{8}$ The general form of this line element is $\left.d s=\left\{\left(a_{i} d x^{i}\right)^{1+\alpha}\left(b_{j} d x^{j}\right)^{1+\beta}\left(c_{k} d x^{k}\right)^{1+\gamma}\left(d_{l} d x^{l}\right)^{1+\delta}\right)\right\}^{1 / 4}$ with $\alpha+\beta+\gamma+\delta=0$. This is an example for the "4th square root of a differential expression of fourth degree" announced by Riemann as the second simplest line element of what later became known as Finsler spaces $[20]$.
} 
a stable degenerate state of space-time and (as a result of the geometric phase transition) may turn either into the partially anisotropic space-time (6) or into the entirely anisotropic space-time (43). Such a phase transition is could be interpreted as an "act of creation" of a 3D space. In the passage to (6) there occurs a 3D space with locally Euclidean geometry while in the passage to (43) there occurs, as will be shown below, a flat 3D space with non-Euclidean geometry. Thus, absolute time plays the role of a connecting link by which a principle of correspondence is satisfied for the Finslerian spaces (6) and (43).

In order to better understand the role of the parameters $r_{1}, r_{2}, r_{3}$, we put in the metric (43) $d x_{2}=d x_{3}=0$. As a result, it turns out that

$$
d s=\left[\left(d x_{0}-d x_{1}\right)^{2} /\left(d x_{0}^{2}-d x_{1}^{2}\right)\right]^{r_{1} / 2} \sqrt{d x_{0}^{2}-d x_{1}^{2}} .
$$

In (43), we now put $d x_{1}=d x_{3}=0$. Then we obtain $d s$ in the form

$$
d s=\left[\left(d x_{0}-d x_{2}\right)^{2} /\left(d x_{0}^{2}-d x_{2}^{2}\right)\right]^{r_{2} / 2} \sqrt{d x_{0}^{2}-d x_{2}^{2}} .
$$

Similarly, by putting in (43) $d x_{1}=d x_{2}=0$, we arrive at the metric

$$
d s=\left[\left(d x_{0}-d x_{3}\right)^{2} /\left(d x_{0}^{2}-d x_{3}^{2}\right)\right]^{r_{3} / 2} \sqrt{d x_{0}^{2}-d x_{3}^{2}} .
$$

Each of these three expressions is idential with the expression (4) which represents the metric of a 2D anisotropic space-time. Therefore, in a sense, the parameters $r_{1}, r_{2}, r_{3}$ characterize the anisotropy along the corresponding axes $x_{1}, x_{2}, x_{3}$. However, space-time (43) is such that it remains anisotropic even at $r_{1}=r_{2}=r_{3}=0$.

In summing up, we see that the $2 \mathrm{D}$ anisotropic metric (4) admits two independent ways of generalization to four dimensions. The first way leads to the partially anisotropic Finslerian 4D metric (6) and the second one to the totally anisotropic Finslerian 4D metric (43).

\section{HOMOGENEOUS GROUP OF RELATIVISTIC SYMMETRY OF THE ENTIRELY ANISOTROPIC SPACE-TIME}

Consider an homogeneous isometry group of the flat space-time (43). By its construction the metric (43) is an invariant of the three independent 1-parameter group of the transformations (39-41). In their infinitesimal form, the transformations belonging to these groups appear as

$$
\begin{gathered}
\left\{\begin{array} { l } 
{ d x _ { 0 } = ( - r _ { 1 } x _ { 0 } - x _ { 1 } ) d \alpha _ { 1 } } \\
{ d x _ { 1 } = ( - r _ { 1 } x _ { 1 } - x _ { 0 } ) d \alpha _ { 1 } } \\
{ d x _ { 2 } = ( - r _ { 1 } x _ { 2 } + x _ { 3 } ) d \alpha _ { 1 } } \\
{ d x _ { 3 } = }
\end{array} \quad \left\{\begin{array}{l}
d x_{0}=\left(-r_{2} x_{0}-x_{2}\right) d \alpha_{2} \\
d x_{1}=\left(-r_{2} x_{1}+x_{3}\right) d \alpha_{2} \\
d x_{2}=\left(-r_{2} x_{2}-x_{0}\right) d \alpha_{2} \\
d x_{3}=\left(-r_{2} x_{3}+x_{1}\right) d \alpha_{2},
\end{array}\right.\right. \\
\left\{\begin{array}{l}
d x_{0}=\left(-r_{3} x_{0}-x_{3}\right) d \alpha_{3} \\
d x_{1}=\left(-r_{3} x_{1}+x_{2}\right) d \alpha_{3} \\
d x_{2}=\left(-r_{3} x_{2}+x_{1}\right) d \alpha_{3} \\
d x_{3}=\left(-r_{3} x_{3}-x_{0}\right) d \alpha_{3} .
\end{array}\right.
\end{gathered}
$$


It can easily be verified that the corresponding generators

$$
\begin{aligned}
& X_{1}=-r_{1} x_{i} \partial / \partial x_{i}-\left(x_{1} \partial / \partial x_{0}+x_{0} \partial / \partial x_{1}\right)+\left(x_{2} \partial / \partial x_{3}+x_{3} \partial / \partial x_{2}\right), \\
& X_{2}=-r_{2} x_{i} \partial / \partial x_{i}-\left(x_{2} \partial / \partial x_{0}+x_{0} \partial / \partial x_{2}\right)+\left(x_{1} \partial / \partial x_{3}+x_{3} \partial / \partial x_{1}\right), \\
& X_{3}=-r_{3} x_{i} \partial / \partial x_{i}-\left(x_{3} \partial / \partial x_{0}+x_{0} \partial / \partial x_{3}\right)+\left(x_{1} \partial / \partial x_{2}+x_{2} \partial / \partial x_{1}\right)
\end{aligned}
$$

commute, i.e. $\left[X_{\alpha} X_{\beta}\right]=0$. It thus appears that the homogeneous 3-parameter noncompact isometry group, i.e. the relativistic symmetry group of the space-time (43) is Abelian and any of its elements can be obtained by multiplying (in an arbitrary order) the transformations (39-41). Having made such multiplication we arrive at the required 3-parameter transformations

$$
x_{i}^{\prime}=D L_{i k} x_{k}
$$

Here $D=\exp \left(-r_{1} \alpha_{1}-r_{2} \alpha_{2}-r_{3} \alpha_{3}\right)$; the matrices

$$
L_{i k}=\left(\begin{array}{rrrr}
\mathcal{A} & -\mathcal{B} & -\mathcal{C} & -\mathcal{D} \\
-\mathcal{B} & \mathcal{A} & \mathcal{D} & \mathcal{C} \\
-\mathcal{C} & \mathcal{D} & \mathcal{A} & \mathcal{B} \\
-\mathcal{D} & \mathcal{C} & \mathcal{B} & \mathcal{A}
\end{array}\right)
$$

are unimodular, whereby

$$
\begin{aligned}
& \mathcal{A}=\cosh \alpha_{1} \cosh \alpha_{2} \cosh \alpha_{3}+\sinh \alpha_{1} \sinh \alpha_{2} \sinh \alpha_{3}, \\
& \mathcal{B}=\cosh \alpha_{1} \sinh \alpha_{2} \sinh \alpha_{3}+\sinh \alpha_{1} \cosh \alpha_{2} \cosh \alpha_{3}, \\
& \mathcal{C}=\cosh \alpha_{1} \sinh \alpha_{2} \cosh \alpha_{3}+\sinh \alpha_{1} \cosh \alpha_{2} \sinh \alpha_{3}, \\
& \mathcal{D}=\cosh \alpha_{1} \cosh \alpha_{2} \sinh \alpha_{3}+\sinh \alpha_{1} \sinh \alpha_{2} \cosh \alpha_{3}
\end{aligned}
$$

and $\alpha_{1}, \alpha_{2}, \alpha_{3}$ are the group parameters. The transformations inverse to (45) can be obtained if we make the substitution

$$
\alpha_{1} \rightarrow-\alpha_{1}, \quad \alpha_{2} \rightarrow-\alpha_{2}, \quad \alpha_{3} \rightarrow-\alpha_{3}
$$

As a result

$$
x_{i}=D^{-1} L_{i k}^{-1} x_{k}^{\prime}
$$

where

$$
L_{i k}^{-1}=\left(\begin{array}{rrrr}
\tilde{\mathcal{A}} & -\tilde{\mathcal{B}} & -\tilde{\mathcal{C}} & -\tilde{\mathcal{D}} \\
-\tilde{\mathcal{B}} & \tilde{\mathcal{A}} & \tilde{\mathcal{D}} & \tilde{\mathcal{C}} \\
-\tilde{\mathcal{C}} & \tilde{\mathcal{D}} & \tilde{\mathcal{A}} & \tilde{\mathcal{B}} \\
-\tilde{\mathcal{D}} & \tilde{\mathcal{C}} & \tilde{\mathcal{B}} & \tilde{\mathcal{A}}
\end{array}\right)
$$

$\tilde{\mathcal{A}}=\cosh \alpha_{1} \cosh \alpha_{2} \cosh \alpha_{3}-\sinh \alpha_{1} \sinh \alpha_{2} \sinh \alpha_{3}$,

$\tilde{\mathcal{B}}=\cosh \alpha_{1} \sinh \alpha_{2} \sinh \alpha_{3}-\sinh \alpha_{1} \cosh \alpha_{2} \cosh \alpha_{3}$,

$\tilde{\mathcal{C}}=\sinh \alpha_{1} \cosh \alpha_{2} \sinh \alpha_{3}-\cosh \alpha_{1} \sinh \alpha_{2} \cosh \alpha_{3}$, 


$$
\tilde{\mathcal{D}}=\sinh \alpha_{1} \sinh \alpha_{2} \cosh \alpha_{3}-\cosh \alpha_{1} \cosh \alpha_{2} \sinh \alpha_{3} .
$$

Since the relativistic symmetry transformations (45) have the same meaning as the Lorentz transformations, it is helpful to use as group parameters, in place of $\alpha_{1}, \alpha_{2}, \alpha_{3}$, the components $v_{1}, v_{2}, v_{3}$ of the velocity of the primed frame. In order to obtain the necessary relations it is sufficient to put $x_{1}^{\prime}=x_{2}^{\prime}=x_{3}^{\prime}=0$ in (46). As a result

$$
v_{1}=\frac{x_{1}}{x_{0}}=-\frac{\tilde{\mathcal{B}}}{\tilde{\mathcal{A}}}, v_{2}=\frac{x_{2}}{x_{0}}=-\frac{\tilde{\mathcal{C}}}{\tilde{\mathcal{A}}}, v_{3}=\frac{x_{3}}{x_{0}}=-\frac{\tilde{\mathcal{D}}}{\tilde{\mathcal{A}}} .
$$

Taking into account (47-50), we can rewrite these formulae as follows

$$
\begin{aligned}
& v_{1}=\left(\tanh \alpha_{1}-\tanh \alpha_{2} \tanh \alpha_{3}\right) /\left(1-\tanh \alpha_{1} \tanh \alpha_{2} \tanh \alpha_{3}\right), \\
& v_{2}=\left(\tanh \alpha_{2}-\tanh \alpha_{1} \tanh \alpha_{3}\right) /\left(1-\tanh \alpha_{1} \tanh \alpha_{2} \tanh \alpha_{3}\right), \\
& v_{3}=\left(\tanh \alpha_{3}-\tanh \alpha_{1} \tanh \alpha_{2}\right) /\left(1-\tanh \alpha_{1} \tanh \alpha_{2} \tanh \alpha_{3}\right) .
\end{aligned}
$$

Now find the inverse relations, i.e. express $\alpha_{1}, \alpha_{2}, \alpha_{3}$ in terms of $v_{1}, v_{2}, v_{3}$. This is easy to do if the following formulae are used

$$
\begin{aligned}
& 1-v_{1}-v_{2}-v_{3}=\frac{\left(1-\tanh \alpha_{1}\right)\left(1-\tanh \alpha_{2}\right)\left(1-\tanh \alpha_{3}\right)}{\left(1-\tanh \alpha_{1} \tanh \alpha_{2} \tanh \alpha_{3}\right)}, \\
& 1-v_{1}+v_{2}+v_{3}=\frac{\left(1-\tanh \alpha_{1}\right)\left(1+\tanh \alpha_{2}\right)\left(1+\tanh \alpha_{3}\right)}{\left(1-\tanh \alpha_{1} \tanh \alpha_{2} \tanh \alpha_{3}\right)}, \\
& 1+v_{1}-v_{2}+v_{3}=\frac{\left(1+\tanh \alpha_{1}\right)\left(1-\tanh \alpha_{2}\right)\left(1+\tanh \alpha_{3}\right)}{\left(1-\tanh \alpha_{1} \tanh \alpha_{2} \tanh \alpha_{3}\right)}, \\
& 1+v_{1}+v_{2}-v_{3}=\frac{\left(1+\tanh \alpha_{1}\right)\left(1+\tanh \alpha_{2}\right)\left(1-\tanh \alpha_{3}\right)}{\left(1-\tanh \alpha_{1} \tanh \alpha_{2} \tanh \alpha_{3}\right)} .
\end{aligned}
$$

As a result we obtain

$$
\begin{aligned}
& \alpha_{1}=\frac{1}{4} \ln \frac{\left(1+v_{1}-v_{2}+v_{3}\right)\left(1+v_{1}+v_{2}-v_{3}\right)}{\left(1-v_{1}-v_{2}-v_{3}\right)\left(1-v_{1}+v_{2}+v_{3}\right)}, \\
& \alpha_{2}=\frac{1}{4} \ln \frac{\left(1-v_{1}+v_{2}+v_{3}\right)\left(1+v_{1}+v_{2}-v_{3}\right)}{\left(1-v_{1}-v_{2}-v_{3}\right)\left(1+v_{1}-v_{2}+v_{3}\right)}, \\
& \alpha_{3}=\frac{1}{4} \ln \frac{\left(1-v_{1}+v_{2}+v_{3}\right)\left(1+v_{1}-v_{2}+v_{3}\right)}{\left(1-v_{1}-v_{2}-v_{3}\right)\left(1+v_{1}+v_{2}-v_{3}\right)} .
\end{aligned}
$$

Since $v_{1}, v_{2}, v_{3}$ by definition are components of the coordinate velocity of the primed frame and the light cone equation for the entirely anisotropic event space (43) differs from the light cone equation of Minkowski space, it is clear that in the entirely anisotropic space an observable such as the magnitude of velocity no longer is determinted by the Euclidean expression $v=\sqrt{v_{1}^{2}+v_{2}^{2}+v_{3}^{2}}$. In order to obtain the correct formula for $v$ it is first necessary to formulate a procedure for synchronizing coordinate clocks, i.e. for determining the difference $\Delta x_{0}$ of the readings of coordinate clocks, which correspond to 
simultaneous events at neighbouring points of the space (43), and also to determine the observable distance between these points.

\section{3D GEOMETRY AND CLOCK SYNCHRONIZATION IN THE ENTIRELY ANISOTROPIC SPACE-TIME}

According to the definition of the totally anisotropic metric (43), the range of permissible values of $d x_{i}$ is limited by the conditions

$$
\left\{\begin{array}{l}
d x_{0}-d x_{1}-d x_{2}-d x_{3} \geq 0 \\
d x_{0}-d x_{1}+d x_{2}+d x_{3} \geq 0 \\
d x_{0}+d x_{1}-d x_{2}+d x_{3} \geq 0 \\
d x_{0}+d x_{1}+d x_{2}-d x_{3} \geq 0
\end{array}\right.
$$

Being invariant under the relativistic transformations (45), these conditions determine either a timelike interval between two events or an interval equal to zero. The latter

case corresponds to events related by a light signal. Owing to the Abelian structure of the group (45) the cited invariance of conditions (52) follows from the relations (39-41). Apart from this, the transformations (45) leave invariant the sign of $d x_{0}$.

Now, let $d x_{0}>0$. Then, in terms of the components $v_{\alpha}=d x_{\alpha} / d x_{0}$ of the coordinate velocity, the conditions (52) can be rewritten as

$$
\left\{\begin{array}{l}
1-v_{1}-v_{2}-v_{3} \geq 0 \\
1-v_{1}+v_{2}+v_{3} \geq 0 \\
1+v_{1}-v_{2}+v_{3} \geq 0 \\
1+v_{1}+v_{2}-v_{3} \geq 0
\end{array} .\right.
$$

The range of $v_{\alpha}$-values, limited by the conditions (53), is represented in Fig. 3 . It forms a regular tetrahedron with its center at the origin $o$ of a rectangular system of coordinates $v_{1}, v_{2}, v_{3}$. The velocities corresponding to the timelike intervals $d s$ fill the inner region of the tetrahedron while the velocities describing the propagation of light signals and guaranteing $d s=0$ fill the surface of the tetrahedron. In comparison, we note that in the case of Minkowski space, in place of (53), the relativistically invariant constraint $1-\boldsymbol{v}^{2} \geq 0$ obtains; i.e., in place of the tetrahedron, a sphere of unit radius as the range of permissible $v_{\alpha}$ values occurs.

Each face of the tetrahedron is described by one of the four equations

$$
\begin{aligned}
& 1-v_{1}-v_{2}-v_{3}=0 \\
& 1-v_{1}+v_{2}+v_{3}=0 \\
& 1+v_{1}-v_{2}+v_{3}=0 \\
& 1+v_{1}+v_{2}-v_{3}=0
\end{aligned}
$$

and each of its six edges by a system of two equations chosen properly from (54-57). The face $\Delta \Xi \Lambda$ is described by Eq. (54), the face $\Psi \Xi \Lambda$, by (55), the face $\Psi \Delta \Xi$, by (56), the 
face $\Psi \Lambda \Delta$, by (57), while for example the edge $\Delta \Xi$, by the system of equations by (54) and (56) etc.

On the surface of the tetrahedron we mark 14 characteristic points: $\alpha, \beta, \gamma, \delta, \epsilon, \zeta, \Gamma$, $\Delta, \Theta, \Lambda, \Xi, \Phi, \Psi, \Omega$. Let us represent the coordinates of these points in the form of the rectangular components of the corresponding radius vectors. In particular: $\overleftarrow{\alpha o}=$ $(1,0,0) ; \overleftarrow{\beta_{O}}=(0,1,0) ; \overleftarrow{\gamma_{O}}=(0,0,1) ; \overleftarrow{\delta_{O}}=(-1,0,0) ; \overleftarrow{\epsilon O}=(0,-1,0) ; \overleftarrow{\zeta_{O}}=$ $(0,0,-1) ; \overleftarrow{\Delta o}=(-1,1,1) ; \overleftarrow{\Lambda_{o}}=(1,-1,1) ; \overleftarrow{\Xi}=(1,1,-1) ;$ and $\overleftarrow{\Psi_{O}}=$ $(-1,-1,-1)$. Similarly the radius vector $\overleftarrow{\Gamma_{o}}=(1 / 3,1 / 3,1 / 3)$ represents the point $\Gamma$. This point is located at the center of the face $\Delta \Xi \Lambda$ and coincides with the projection of the vertex $\Psi$ onto this face. The radius vector $\widetilde{\Omega_{0}}=(1 / 3,-1 / 3,-1 / 3)$ represents the point $\Omega$ located at the center of the face $\Psi \Xi \Lambda$ and coinciding with the projection of the vertex $\Delta$ onto it. Likewise, the radius vector $\overleftarrow{\Phi_{O}}=(-1 / 3,1 / 3,-1 / 3)$ represents the point $\Phi$ located at the center of the face $\Psi \Delta \Xi$ and coinciding with the projection of the vertex $\Lambda$ onto this face. Finally, the radius vector $\overleftarrow{\Theta_{o}}=(-1 / 3,-1 / 3,1 / 3)$ represents the point $\Theta$ located at the center of the face $\Psi \Lambda \Delta$ and coinciding with the projection of the vertex $\Xi$ onto this face.

By these characteristic points on the tetrahedron surface this surface is divided into twelve equal tetragons which, in turn, are grouped into six pairs of mutually conjugate ${ }^{\text {g }}$ tetragons. Denoting the reflection operation by a symbol $\longleftrightarrow$, we obtain the following pairs

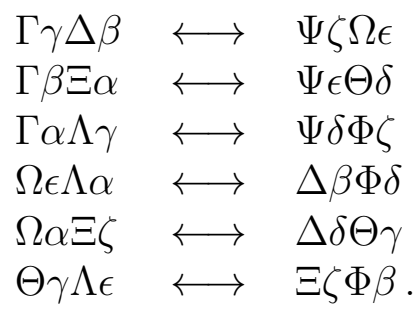

In accordance with the division of the tetrahedron surface, the full solid angle $4 \pi$ is also divided into six pairs of mutually conjugate sectors. Each of these sectors constitutes a tetrahedral solid angle which rests on the corresponding tetragon and has its vertex at the origin of the coordinates. Consider, for example, the sector $\Gamma \gamma \Delta \beta o$ which rests on the tetragon $\Gamma \gamma \Delta \beta$. This tetragon belongs to the face $\Delta \Xi \Lambda$. Therefore, the coordinates $v_{1}, v_{2}, v_{3}$ of any inner point of such a tetragon or of a point belonging to its boundary satisfy Eq. (54), in which case the radius vector $\overleftarrow{v o}=\left(v_{1}, v_{2}, v_{3}\right)$ represents the coordinate velocity of an initial light ray propagating within the sector $\Gamma \gamma \Delta \beta o$ since $d s=0$ (in virtue of (54)). Using Fig. 3, and taking into consideration the equation

$$
1-\tilde{v}_{1}+\tilde{v}_{2}+\tilde{v}_{3}=0
$$

which describes the face $\Psi \Xi \Lambda$, ( cf. (55)) it is easy to verify that the radius vector $\tilde{\widetilde{v o}}=$ $\left(\tilde{v}_{1}, \tilde{v}_{2}, \tilde{v}_{3}\right)$ with components

$$
\tilde{v}_{1}=-\frac{v_{1}}{v_{2}+v_{3}-v_{1}} ; \quad \tilde{v}_{2}=-\frac{v_{2}}{v_{2}+v_{3}-v_{1}} ; \quad \tilde{v}_{3}=-\frac{v_{3}}{v_{2}+v_{3}-v_{1}},
$$

\footnotetext{
${ }^{9}$ with respect to a reflection operation at the origin
} 
where, according to (54),

$$
v_{1}+v_{2}+v_{3}=1
$$

represents the coordinate velocity of a reflected light ray. Compared with the initial ray such a ray has the opposite direction and propagates within the sector $\Psi \zeta \Omega \epsilon o$ which rests on the tetragon $\Psi \zeta \Omega \epsilon$. Formulae (59), (60) give a one-to-one mapping of the tetragon $\Gamma \gamma \Delta \beta$ onto the tetragon $\Psi \zeta \Omega \epsilon$. The formulae inverse to (59), (60) appear as

$$
v_{1}=\frac{\tilde{v}_{1}}{\tilde{v}_{1}+\tilde{v}_{2}+\tilde{v}_{3}} ; v_{2}=\frac{\tilde{v}_{2}}{\tilde{v}_{1}+\tilde{v}_{2}+\tilde{v}_{3}} ; v_{3}=\frac{\tilde{v}_{3}}{\tilde{v}_{1}+\tilde{v}_{2}+\tilde{v}_{3}},
$$

where, according to (58),

$$
\tilde{v}_{2}+\tilde{v}_{3}-\tilde{v}_{1}=-1
$$

These formulae also give a one-to-one mapping of the tetragon $\Psi \zeta \Omega \epsilon$ onto the tetragon $\Gamma \gamma \Delta \beta$. It is precisely in connection with the mappings (59), (60) and (61), (62) that the tetragons $\Gamma \gamma \Delta \beta$ and $\Psi \zeta \Omega \epsilon$ (as well as the corresponding sectors) were called mutually conjugate above.

The formulae, which relate the components of the coordinate velocities of initial and reflected light rays are modified in the passage from one pair of mutually conjugate sectors to another. This involves a corresponding change in the formulae for the observables, the change being such that the observables remain continuous at the boundaries separating neighbouring sectors. This is confirmed by the Table given below, in which formulae are collected which determine the observables for each of the twelve sectors. For illustrative purposes we reproduce here only the formulae pertaining to the sector $\Gamma \gamma \Delta \beta o$. The meaning of all the symbols involved in the Table of observables should now have become obvious.

In order to determine how the difference of the coordinates of two events in the event space (43) is correlated with the observables, we use the Einstein procedure implying exchange of light signals between points of 3D space.

Let an initial event $I$, with coordinates $(0,0,0,0)$, be involved in the emission of a light signal, and another event $R$ with coordinates $\left(d x_{0}^{(1)}, d x_{1}, d x_{2}, d x_{3}\right)$ be involved in the reflection of this signal. In addition, let the $d x_{1}, d x_{2}, d x_{3}$ be such that the initial signal propagates within the sector $\Gamma \gamma \Delta \beta o$. Represent the components of the coordinate velocity of the initial signal in the form

$$
v_{1}=\frac{d x_{1}}{d x_{0}^{(1)}} ; \quad v_{2}=\frac{d x_{2}}{d x_{0}^{(1)}} ; \quad v_{3}=\frac{d x_{3}}{d x_{0}^{(1)}} .
$$

Finally, let

$$
\left(d x_{0}^{(1)}+d x_{0}^{(2)}, 0,0,0\right)
$$

be the coordinates of a final event $F$, involving the return of the signal to the initial point after its reflection. Represent the components of the coordinate velocity of the reflected signal in the form

$$
\tilde{v}_{1}=-\frac{d x_{1}}{d x_{0}^{(2)}} ; \quad \tilde{v}_{2}=-\frac{d x_{2}}{d x_{0}^{(2)}} ; \quad \tilde{v}_{3}=-\frac{d x_{3}}{d x_{0}^{(2)}}
$$


It was mentioned before that the reflected signal propagates within the sector $\Psi \zeta \Omega \epsilon o$ conjugate to the sector $\Gamma \gamma \Delta \beta o$. It is therefore clear from Fig. 3 that $\tilde{v}_{\alpha} \neq-v_{\alpha}$ and, consequently, $d x_{0}^{(2)} \neq d x_{0}^{(1)}$. In virtue of (65), (63), (59), we have

$$
\begin{array}{r}
-v_{1} / \tilde{v}_{1}=-v_{2} / \tilde{v}_{2}=-v_{3} / \tilde{v}_{3}= \\
d x_{0}^{(2)} / d x_{0}^{(1)}=v_{2}+v_{3}-v_{1} .
\end{array}
$$

The latter equality, together with (60), makes up a system of two equations. It will be written as

$$
\left\{\begin{array}{l}
d x_{0}^{(2)} / d x_{0}^{(1)}=2\left(v_{2}+v_{3}\right)-1 \\
d x_{0}^{(2)} / d x_{0}^{(1)}=1-2 v_{1} .
\end{array}\right.
$$

Hence, taking into account (63), we obtain the following relations

$$
\begin{gathered}
\left(d x_{0}^{(1)}+d x_{0}^{(2)}\right) / 2=d x_{2}+d x_{3}, \\
\left(d x_{0}^{(1)}-d x_{0}^{(2)}\right) / 2=d x_{1} .
\end{gathered}
$$

Turning to the definition (64), it is easily understood that the quantity $\left(d x_{0}^{(1)}+\right.$ $\left.d x_{0}^{(2)}\right) / 2$ prescribes the $3 \mathrm{D}$ distance $d l$ between the events $I$ and $R$. By definition, these events have 3D coordinates $(0,0,0)$ and $\left(d x_{1}, d x_{2}, d x_{3}\right)$, respectively, in which case the vector $d \boldsymbol{x}=\left(d x_{1}, d x_{2}, d x_{3}\right)$ falls into the sector $\Gamma \gamma \Delta \beta o$. Thus, within the given sector, the relation (66) gives

$$
d l=d x_{2}+d x_{3} .
$$

We now consider a procedure which allows synchronization of coordinate clocks (i.e. clocks reading the coordinate time $x_{0}$ ) located at the neighbouring points $\mathcal{I}$ and $\mathcal{R}$ of 3D space; we intend to determine the difference $\Delta x_{0}$ between the readings of these neighbouring clocks, which corresponds to simultaneous events at $\mathcal{I}$ and $\mathcal{R}$.

Let $(0,0,0)$ and $\left(d x_{1}, d x_{2}, d x_{3}\right)$ be the 3D coordinates of the points $\mathcal{I}$ and $\mathcal{R}$, respectively. Choose as one of the events the event $R$ at the point $\mathcal{R}$ which has the coordinates $\left(d x_{0}^{(1)}, d x_{1}, d x_{2}, d x_{3}\right)$. Then another event $S$ at the point $\mathcal{I}$, with coordinates $\left(\left(d x_{0}^{(1)}+d x_{0}^{(2)}\right) / 2,0,0,0\right)$ is obviously simultaneous to the event $R$ at the point $\mathcal{R}$. As a result

$$
\Delta x_{0}=d x_{0}^{(1)}-\frac{d x_{0}^{(1)}+d x_{0}^{(2)}}{2}=\frac{d x_{0}^{(1)}-d x_{0}^{(2)}}{2} .
$$

Using the relation (67), we finally find that

$$
\Delta x_{0}=d x_{1}
$$

This formula permits synchronization of clocks within the sector $\Gamma \gamma \Delta \beta o$.

Moreover, consider the motion of a particle and determine $v$, i.e. the observable values of its velocity. For obtaining $v$, it is necessary first to know the true time $d \tau$, spent by this particle on the displacement $d \boldsymbol{x}=\left(d x_{1}, d x_{2}, d x_{3}\right)$.

If the particle starts from point $\mathcal{I}$ at an instant of coordinate time 0 and reaches point $\mathcal{R}$ at an instant of coordinate time $d x_{0}$, then the true time $d \tau$ spent on the displacement 
is not equal to $d x_{0}$ but equal to the difference between the instants $d x_{0}$ and $\Delta x_{0}$ which is simultaneous at $\mathcal{R}$ to the instant 0 at the starting point $\mathcal{I}$, i.e. $d \tau=d x_{0}-\Delta x_{0}$. Thus, using (69), we get

$$
d \tau=d x_{0}-d x_{1} .
$$

As a result, from (68) and (70)

$$
v=\frac{d l}{d \tau}=\frac{d x_{2}+d x_{3}}{d x_{0}-d x_{1}}=\frac{v_{2}+v_{3}}{1-v_{1}} .
$$

The formula given shows how within the sector $\Gamma \gamma \Delta \beta o$ the observable value of the particle velocity is expressed in terms of the components $v_{1}, v_{2}, v_{3}$ of its coordinate velocity. According to (71), $v \leq 1$, with $v=1$ for a photon. In the latter case, (71) is equivalent to (54) and thus to the light cone equation $d s=0$.

After similar calculations for each of the remaining eleven sectors we obtain the complete set of formulae which determine the observables. These formulae are tabulated in the Table of the observables. According to this Table, the symmetry of 3D space is determined not by the rotation group but by a corresponding group of discrete transformations: the flat 3D space which corresponds to the totally anisotropic event space (43) is non-Euclidean. This is demonstrated most easily if we graphically reproduce an Euclidean image of the sphere of radius $d l$ prescribed in the flat non-Euclidean 3D space. For this purpose, a rectangular system of coordinates $d x_{1}, d x_{2}, d x_{3}$ is introduced in Euclidean 3D space and use is made of the relations presented in the second column of the Table. It can readily be seen that each of the twelve sectors cuts its own piece (a rhomb) out of the corresponding plane $d l=$ const. All twelve rhombs turn out to be equal to each other and taken together constitute the surface of a regular rhombic dodecahedron. Such a dodecahedron is illustrated in Fig. 4. The Cartesian coordinates of 14 vertices of the dodecahedron are represented as rectangular components of the corresponding radius vector

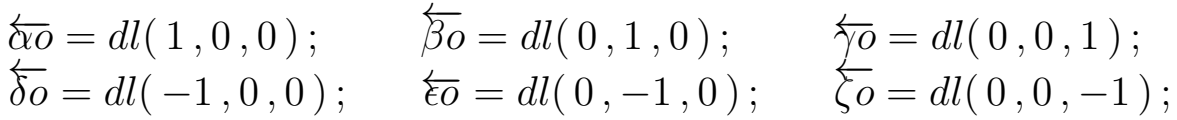

$$
\begin{aligned}
& \overleftarrow{\Gamma o}=d l(1 / 2,1 / 2,1 / 2) ; \quad \overleftarrow{\Delta o}=d l(-1 / 2,1 / 2,1 / 2) ; \\
& \overleftarrow{\Theta o}=d l(-1 / 2,-1 / 2,1 / 2) ; \quad \overleftarrow{\Lambda_{o}}=d l(1 / 2,-1 / 2,1 / 2) \text {; } \\
& \overleftarrow{\exists o}=d l(1 / 2,1 / 2,-1 / 2) ; \quad \overleftarrow{\Phi o}=d l(-1 / 2,1 / 2,-1 / 2) \text {; } \\
& \overleftarrow{\Psi o}=d l(-1 / 2,-1 / 2,-1 / 2) ; \quad \overleftarrow{\Omega_{o}}=d l(1 / 2,-1 / 2,-1 / 2) \text {. }
\end{aligned}
$$

By the coordinates of the vertices it is easy to calculate an acute angle of any rhomb, e.g. $\angle \Gamma \gamma \Delta$. It turns out that

$$
\angle \Gamma \gamma \Delta=\arccos \frac{1}{3} \approx 70^{\circ} .
$$

Needless to say that in comparison with Minkowski space the relativistically invariant Finslerian space-time (43) -with entirely broken isotropy of 3D space- possesses more exotic properties than the relativistically invariant Finslerian space-time (6) with partially broken isotropy. In spite of the fact that, proceeding from the flat metric (43), it is easy 
to build the corresponding model of a curved Finslerian space possessing local relativistic symmetry and local entire 3D anisotropy, it is still diffucult to indicate the place which such a model could occupy in modern physics.t?

\section{A FIELD OF LOCAL ANISOTROPY AND THE FINSLERIAN MODEL OF A CURVED SPACE-TIME}

It is obvious that within the framework of the model of flat Finslerian spaces as given by (6) or (43), it is impossible to answer constructively the question of the possible origin of local anisotropy. While discussing the physical nature of inertia, Mach arrived at the conclusion that it is unreasonable to speak of the acceleration of a body relative to empty space. Inertia of bodies should be regarded as their ability to resist acceleration relative to external matter. Since external matter is distributed nonuniformly, inertia and inertial forces arising from acceleration should depend on the localization of a body and on the direction of its acceleration. Consequently, inertial mass should be a quantity represented by a tensor field over space-time. When this conclusion is compared with the fact that inertial mass in anisotropic space is represented by a tensor, such a comparison suggests that the parameters $r$ and $\boldsymbol{\nu}$, in terms of which the inertial mass (29) is expressed, should be regarded not as constants but as fields over space-time with a matter distribution as their source. Consequently, we should also consider a space-time with local anisotropy varying from point to point. Then, due to the dependence on the fields $r$ and $\boldsymbol{\nu}$ characterizing the local anisotropy of what will turn out to be a curved space-time, the inertial mass (29) will acquire the character of a tensor field in correspondence with Mach's principle. In relativistic metric theories of gravitation, where $r=0$, such a result cannot be obtained.

The Finslerian metric of a curved locally anisotropic space-time must be of such a form that, on the one side, the principle of correspondence with the Riemannian metric of a curved locally isotropic space-time of GR is satisfied, and on the other side, at any point it ought to admit a representation in the form (6) П. The Finslerian metric with the above-mentioned properties turns out to be the following

$$
d s=\left[\frac{\left(\nu_{i} d x^{i}\right)^{2}}{g_{i k} d x^{i} d x^{k}}\right]^{r / 2} \sqrt{g_{i k} d x^{i} d x^{k}} .
$$

The given metric is a function of three fields: $r=r(x)$, a scalar field determining the magnitude of local anisotropy; $\nu_{i}=\nu_{i}(x)$, a vector field of locally preferred directions in space-time satisfying the condition $\nu_{i} \nu^{i}=g_{i k} \nu^{i} \nu^{k}=0$, and finally $g_{i k}=g_{i k}(x)$, the field of a Riemannian metric tensor. At each of its points, the curved Finslerian space-time (72) has its own tangent space (6) with its own values of the parameters $r$ and $\boldsymbol{\nu}$ which

\footnotetext{
${ }^{10} \mathrm{~A}$ possible speculation would be that the flat Finslerian metric (43) describe the space geometry in the asymptotically free limit of quantum chromodynamics, i.e. at distances much smaller than the tenth part of a fermi.

${ }^{11}$ or $(43)$
} 
determine the local anisotropy. These values of the parameters are none other than the local values of the corresponding fields $r(x)$ and $\nu_{i}(x)$.

The metric (72) is written in arbitrary coordinates. It is therefore important to elucidate how the difference of the coordinates of two neighbouring events is related to observables. First of all consider proper time. From (72) the interval $d \tau$, measured by an observer at rest at a point with spatial coordinates $x^{\alpha}$, is related to the interval $d x^{0}$ of coordinate time by the relation $c d \tau=\left(\nu_{0}^{2} / g_{00}\right)^{r / 2} \sqrt{g_{00}} d x^{0}$. For obtaining the 3D distance between neighbouring points and for synchronizing the coordinate clocks it is necessary to use the exchange of light signals. This can easily be done since the light cone equation remains the same as in GR (in accordance with (72)). As a result, the 3D metric turns out to be the following: $d l^{2}=\gamma_{\alpha \beta} d x^{\alpha} d x^{\beta}$, where $\gamma_{\alpha \beta}=\left(\nu_{0}^{2} / g_{00}\right)^{r}\left(-g_{\alpha \beta}+g_{0 \alpha} g_{0 \beta} / g_{00}\right)$ and the difference $\Delta x^{0}$ of the readings of the coordinate clocks recording the simultaneous events at the neighbouring points is given by the formula $\Delta x^{0}=-g_{0 \alpha} d x^{\alpha} / g_{00}$.

The structure of the locally anisotropic Finslerian space (72) is such that the motions of massless particles and of test bodies in it are significantly different. Light propagates along Riemannian geodesics with the metric tensor $g_{i k}$ whereas free fall of test bodies occurs along Finslerian geodesics [33].

According to (72), the dynamics of Finslerian space-time is completely determined by the dynamics of the gravitational field $g_{i k}(x)$ and of the fields $r(x)$ and $\nu_{i}(x)$, responsible for local anisotropy. Since these three fields interact with each other and with matter, for a description of the dynamics it is necessary to construct equations which generalize the corresponding Einstein equations. The key role in solving this task is played by the property of invariance of the Finslerian metric (72) under the transformations

$$
g_{i k} \rightarrow e^{2 \sigma(x)} g_{i k}, \quad \nu_{i} \rightarrow e^{(r-1) \sigma(x) / r} \nu_{i},
$$

where $\sigma(x)$ is an arbitrary function. Apart from the metric, the local transformations (73) leave invariant all the observables. Therefore in the theory taking account of the anisotropy of space-time the transformations (73) are local gauge transformations. Gaugeinvariant, for example, is the action for a compressible fluid in the Finslerian space [34]

$$
S=-\frac{1}{c} \int \mu^{*}\left(\frac{\nu_{i} v^{i}}{\sqrt{g_{i k} v^{i} v^{k}}}\right)^{4 r} \sqrt{-g} d^{4} x,
$$

where $\mu^{*}$ is the invariant fluid energy density, $v^{i}=d x^{i} / d s$, and $d s$ is the Finslerian metric $(72)$.

In connection with the mentioned gauge invariance, the dynamic system consisting of the fields $g_{i k}, r, \nu_{i}$ and a compressible fluid must be complemented with a vector gauge field $B_{i}$ which under (73) transforms as follows

$$
B_{i} \rightarrow B_{i}+b[(r-1) \sigma(x) / r]_{; i},
$$

where $b$ is a constant with a dimension of length. As a result, the behaviour of the given system is described by the following gauge-invariant variational principle

$$
\delta \int\left\{-\frac{1}{2}[\cdots] R-\frac{3}{4}[\cdots]^{-1}[\cdots]^{; i}[\cdots]_{; i}-\frac{r^{; i} r_{; i}}{4 \varsigma(\varepsilon-r)}\left(\frac{\cdots}{\cdots}\right)^{2 r}\right.
$$




$$
\begin{gathered}
-\frac{f}{4} \mathcal{N}_{i k} \mathcal{N}^{i k}\left(\frac{\cdots}{\cdots}\right)^{2 r-2}+\frac{1}{2} \lambda^{2} f \nu_{i} \nu^{i}\left(\frac{\cdots}{\cdots}\right)^{4 r-2}-\frac{1}{4} \mathcal{F}_{i k} \mathcal{F}^{i k} \\
\left.-\frac{8 \pi \hat{k}}{c^{4}} \mu^{*}\left(\frac{\nu_{i} v^{i}}{\sqrt{g_{i k} v^{i} v^{k}}}\right)^{4 r}\right\} \sqrt{-g} d^{4} x=0
\end{gathered}
$$

where $\left(\frac{\cdots}{\cdots}\right)=\left(\nu^{k} r_{; k} / \sqrt{-r_{; k} r^{; k}}\right), \quad[\cdots]=\left[(1-r / \varepsilon)\left(\frac{\cdots}{\cdots}\right)^{2 r}\right], \quad \mathcal{N}_{i k}=\nu_{k ; i}-\nu_{i ; k}-\left(\nu_{k} B_{i}-\right.$ $\left.\nu_{i} B_{k}\right) / b, \quad \mathcal{F}_{i k}=B_{k ; i}-B_{i ; k}, R$ is a Riemannian scalar. The constants $f, \varsigma$ and $1 / \varepsilon$ are dimensionless; $\varsigma$ characterizes the interaction of the fluid (matter) with the field $r$ while $1 / \varepsilon$, the interaction of the fields $r$ and $g_{i k} ; \hat{k}$ is a gravitational constant related to the observable Newtonian constant by $\hat{k}=k / \eta ; \eta$ is a renormalization constant given by the formula

$$
\eta=1+\frac{\varsigma /(2 \varepsilon)}{[1+\varsigma /(4 \varepsilon)]^{1 / 2}}
$$

and, finally, $\lambda^{2}$ is a Lagrange multiplier.

The variational principle (74) leads to the equations of relativistic hydrodynamics in the locally anisotropic space and also to a system of gauge-invariant field equations. In a gauge given by the condition $\nu^{k} r_{; k}=\sqrt{-r_{; k} r^{; k}}$, the corresponding system of field equations is presented in $[12,13]$. It should be noted that if the existence of a "fifth force" is confirmed then the gauge field $B_{i}$ may be regarded as its carrier. An additional term $\sim B_{i} j^{i}$ must then be incorporated in the variational principle (74), where $j^{i}$ is a preserved current involved in the hydrodynamic equations $\square$.

In ref. [11], the static centrally symmetric solution of the new field equations was found, i.e. the Finslerian problem of Schwarzschild solved. Subsequently, in a post-Newtonian approximation the equations of Finslerian geodesics were integrated and corrections to the classical gravitational effects arising from the local anisotropy of space-time were calculated. Comparison of these corrections with error estimates in the experimental data relating to the solar system gives the following constraints on the interaction constants

$$
-0,054<\varsigma \leq 0, \quad 0<1 / \varepsilon<0,25 .
$$

Within the framework of the Finslerian theory, the equality $\varsigma=0$ means the absence of the field $r$ determining the magnitude of the local anisotropy of space-time. In this case the Finslerian metric (72) reduces to the Riemannian one and the Finslerian gravitation theory to the Einstein theory. If $\varsigma \neq 0$, the presence of the field of locally preferred directions introduces a partial ordering into the structure of space-time; it is precisely by this that the Finslerian space-time (72) is distinguished from the "amorphous" Riemannian space. It must be added here that, according to the field equations of the Finslerian theory, the main source of the field $r$ is the trace of the energy-momentum tensor for the matter fields which is zero for the massless and nonzero for massive fields. As a result, a scenario of the evolution of the Universe becomes possible where only initially, i.e. before the appearance of high-temperature phase transitions with a successive breaking of

\footnotetext{
${ }^{12}$ Such a refinement of the variational principle (74) actually seems to be necessary. In this connection see the report [35].
} 
higher gauge symmetries and before the appearance of masses in the fundamental matter field, space-time was Riemannian. With the appearance of massive elementary particles, the trace of the energy-momentum tensor becomes nonzero. In this case, a strong local anisotropy of space-time is generated, i.e. there occur phase transitions in its local geometric structure as a result of which space-time acquires a Finslerian metric. In the course of the subsequent expansion the initially strong local anisotropy of space gradually decreases and on the average tends to zero along with its curvature. Thereby, the Finslerian space-time again approximates a Riemannian one. Apparently, it is the induced phase transitions in the geometric structure of space-time which make energetically most favourable the scheme of breaking higher gauge symmetries realized in nature.

\section{CONCLUSION}

We descibed two types of Finslerian event spaces, namely, spaces with partially and entirely broken local rotational symmetry in 3D space. Since the locally isotropic Riemannian space-time is a special case of the Finslerian space-time (72) (corresponding to the vanishing of the field $r$ ), one can speak of a joint description of three geometric models of space-time. It is important to stress that each of the above-mentioned models possesses (differing) local relativistic invariances. Depending on the magnitude and character of the breaking of local 3D isotropy, local relativistic invariance may take either the form of full Lorentz invariance (3D rotational symmetry not broken), the form of generalized Lorentz invariance, i.e. invariance under the transformations (7) (partial breaking of isotropy), or invariance under the transformations (45) (total breaking of isotropy).

Experimental discoveries of recent years, in particular the discovery of the anisotropy of the cosmic background radiation have led to a renaissance of interest in theories with a preferred frame of reference. In essence, in such investigations, the old idea of an absolute "ether" is exploited, the only difference being that the preferred frame is now identified with a frame in which the cosmic background radiation is locally isotropic and the already established physical laws are operative. In this case, attempts are sometimes undertaken to explain new experimental results by an ad-hoc breaking of Lorentz invariance in the passage from the preferred frame to another (laboratory) inertial frame. In this way, certainly "anything" can be "explained". At the same time, Einstein's principle of relativity, implemented with help of the generalized Lorentz transformations, allows the avoidance of such a diversity of options, and convincingly leads to local anisotropy of space-time. As a result, the problem of a possible violation of the Lorentz transformations reduces to the problem of existence of local anisotropy of space-time.[3]

In connection with a possible local anisotropy of space-time it will be recalled that according to the model of the hot Universe the temperature of relic radiation should not depend on the direction in which it is being measured. At the same time the temperature anisotropy of relic radiation is already an experimental fact with dipole component of

\footnotetext{
${ }^{13}$ A different approach to deviations from Lorentz invariance which, however, leads to a more complicated physics was followed in refs. [36].
} 
anisotropy having the largest value. Investigators usually do not express a fundamental interest in such a dipole anisotropy because they believe that it arises from the fact that our lab frame accidentally moves at a certain velocity relative to the cosmic microwave background. Such an explanation would be more satisfactory if the corresponding anisotropy were also observed in the Hubble constant. Until now, studies of the angular dependence of the Hubble constant are neither precise enough nor covering a larger section of the sky. ( Cf. [37].) If a special analysis will show that there is no correlated dipole anisotropy in the Hubble constant then the dipole anisotropy of relic radiation might be an indication of a strong local anisotropy of space-time at an early stage of the evolution of the Universe. The point is that in a space with strong anisotropy there indeed exists a physically preferred frame; with respect to this frame the hot background radiation was isotropic while the velocity distribution of massive relativistic particles was anisotropic. As a result, the Hubble constant became anisotropic. Therefore, by passage to another frame, a reversed situation becomes possible: the Hubble constant looses its dipole anisotropy while the background radiation picks it up.

It has already been noted that the experimental data on the behaviour of the spectra of primary ultra-high energy cosmic protons were one of the motivations for the Finslerian generalization of relativity theory. In spite of indirect evidence in favour of it, the relativistic theory of locally anisotropic space-time, outlined in the present paper, is still in need of empirical support. Since the alternative to local anisotropy is a strict local isotropy of space-time, and since in nature any strict symmetry holds only approximately, it seems reasonable to continue investigations into the physical manifestations of local anisotropy. In fact, such a line of research is equivalent to the testing of SR and of Lorentz invariance to which increased attention has been paid recently, both from the experimental [38] and theoretical side [39]. 


\section{REFERENCES}

1. Rund, H. (1959). The Differential Geometry of Finsler Spaces (Springer, Berlin).

2. Doi, T., et al. (1995). In Proc. 24th Int. Cosmic Ray Conf., 2 (Roma),740.

3. Greisen, K. (1966). Phys. Rev. Lett. 16, 748.

4. Zatsepin, G. T., and Kuz'min, V. A. (1966). Pis'ma Zh. Eksper. Teor. Fiz. 4, 114.

5. Kirzhnits, D. A., and Chechin, V. A. (1971). Pis'ma Zh. Eksper. Teor. Fiz.

14, 261.

6. Kirzhnits, D. A., and Chechin, V. A. (1972). Yadern. Fiz. 15, 1051.

7. Khristiansen, G. B. (1974). Cosmic Rays of Superhigh Energies (Moscow Univ. Press, Moscow), in Russian.

8. Coleman, S., and Glashow, S. L. (1998). Preprint hep-ph/9808446.

9. Berezinsky, V. S. (1998). Preprint INFN/TH-98/03.

10. Bogoslovsky, G. Yu. (1977). Nuovo Cimento B40, 99.

11. Bogoslovsky, G. Yu. (1992). Theory of Locally Anisotropic Space-Time (Moscow Univ. Press, Moscow), in Russian.

12. Bogoslovsky, G. Yu. (1992). Class. Quantum Grav. 9, 569.

13. Bogoslovsky, G. Yu. (1994). Fortschr. Phys. 42, 143; (1993). Phys. Part. Nucl. 24, 354 .

14. Cunningham, E. (1910). Proc. London Math. Soc. 8, 77.

15. Bateman, H. (1910). Proc. London Math. Soc. 8, 223.

16. Fulton, T., Rohrlich, F., and Witten, L. (1962). Rev. Mod. Phys. 34, 442.

17. Bogoslovsky, G. Yu., and Goenner, H. F. (1995). In Abstr. Int. School-Seminar "Found. of Grav. and Cosmology", (Odessa), 79.

18. Bogoslovsky, G. Yu., and Goenner, H. F. (1997). In Abstr. 5th Int. Wigner Symposium, (Vienna), 20.

19. Bogoslovsky, G. Yu., and Goenner, H. F. (1998). Phys. Lett. A244, 222.

20. Riemann, B. (1867). Abhandlungen der Königl. Gesellschaft d. Wissenschaften, Göttingen 13, 14. 
21. Winternitz, P., and Friš, I. (1965). Yadern. Fiz. 1, 889.

22. Goenner, H. F., and Bogoslovsky, G. Yu. (1997). Preprint gr-qc/9701067; Submitted to Gen. Rel. Grav.

23. Tavakol, R. K., and Van den Bergh, N. (1985). Phys. Lett. A112, 23.

24. Tavakol, R. K., and Van den Bergh, N. (1986). Gen. Rel. Grav. 18, 849.

25. Bogoslovsky, G. Yu. (1976). Pis'ma Zh. Eksper. Teor. Fiz. 23, 192.

26. Møller, C. (1962). Proc. R. Soc. A207, 306.

27. Champeney, D. C., Isaak, G. R., and Khan, A. M. (1963). Phys. Lett. 7, 241.

28. Kaivola, M., Poulsen, A., et al. (1985). Phys. Rev. Lett. 54, 255;

McGowan, R. W., Giltner, D. M., et al. (1993). Phys. Rev. Lett. 70, 251.

29. Isaak, G. R. (1970). Phys. Bull. 21, 255.

30. Bogoslovsky, G. Yu. (1977). Nuovo Cimento B40, 116.

31. Barbour, J. B. (1974). Nature, 328; (1975). Nuovo Cimento B26, 16;

Barbour, J. B., and Bertotti, B. (1977). Nuovo Cimento B38, 1.

Cf. the review of Goenner, H. (1981). In Grundlagenprobleme der modernen Physik,

Eds. Nitsch, J., et al. (Bibliographisches Institut, Mannheim), 85.

32. Noether, E. (1918) Göttinger Nachrichten. Math. Phys. Kl H2, 235.

33. Bogoslovsky, G. Yu. (1984). Ukr. Fiz. Zh. 29, 17.

34. Bogoslovsky, G. Yu. (1986). Dokl. Akad. Nauk SSSR 291, 317.

35. Anderson, J. D., et al. (1998). Phys. Rev. Lett. 81, 2858.

36. Bleyer, U., and Liebscher, D. E. (1986). Astronomische Nachrichten 307, 267;

Bleyer, U. (1988). In Gravitation und Kosmos, Ed. Wahsner, R.

(Akademie-verlag, Berlin), 91.

37. Ichikawa, T., and Fukugita, M. (1992). Astrophysical Journal 394, 66.

38. Nielsen, H. B., and Picek, I. (1982). Phys. Lett. B114, 141;

Fischbach, E., Haugan, M. P., Tadic, D., and Hai-Yang-Cheng. (1985). Phys. Rev. D32, 154 .

39. Mansouri, R., and Sexl, R. U. (1977). Gen. Rel. Grav. 8, 497; 515;

Golestanian, R., Khajehpour, M. R. H., and Mansouri, R. (1995). Class. Quantum Grav. 12, 273. 


\section{TABLE AND FIGURE CAPTIONS}

Tabl. I : Table of observables.

Fig. 1 : Plots for $d \tau / d t=\left[(1-v \cos \alpha / c) / \sqrt{1-v^{2} / c^{2}}\right]^{r} \sqrt{1-v^{2} / c^{2}}$ at $r=0.6$ and at three successive values $0, \pi / 4, \pi / 2$ of the angle $\alpha$ between $\boldsymbol{v}$ and $\boldsymbol{\nu}$. These plots demonstrate the specific features of the behaviour of the anisotropic factor of time dilatation $\left.(d \tau / d t)\right|_{r>0}$ in comparison with the behaviour of the isotropic (Minkowskian) factor $\left.(d \tau / d t)\right|_{r=0}$.

Fig. 2 : Parametric 3D plots illustrating the dependence of deformation of a two-sheet hyperboloid on the magnitude $r$ of space anisotropy. Any of the deformed hyperboloids remains inscribed into a light cone and like a light cone it is an invariant of the generalized Lorentz transformations (22).

Fig. 3 : The relativistically invariant range of permissible $v_{\alpha}$ values.

Fig. 4: A regular rhombic dodecahedron as an Euclidean image of the sphere of radius $d l$, prescribed in the flat non-Euclidean 3D space. 
Table of observables

\begin{tabular}{|c|c|c|c|}
\hline sector & $d l$ & $\Delta x_{0}$ & $v$ \\
\hline$\Gamma \gamma \Delta \beta o$ & $d x_{2}+d x_{3}$ & $d x_{1}$ & $\left(v_{2}+v_{3}\right) /\left(1-v_{1}\right)$ \\
\hline$\Psi \zeta \Omega \epsilon O$ & $-\left(d x_{2}+d x_{3}\right)$ & $d x_{1}$ & $-\left(v_{2}+v_{3}\right) /\left(1-v_{1}\right)$ \\
\hline$\Gamma \beta \Xi \alpha O$ & $d x_{1}+d x_{2}$ & $d x_{3}$ & $\left(v_{1}+v_{2}\right) /\left(1-v_{3}\right)$ \\
\hline$\Psi \epsilon \Theta \delta o$ & $-\left(d x_{1}+d x_{2}\right)$ & $d x_{3}$ & $-\left(v_{1}+v_{2}\right) /\left(1-v_{3}\right)$ \\
\hline$\Gamma \alpha \Lambda \gamma o$ & $d x_{1}+d x_{3}$ & $d x_{2}$ & $\left(v_{1}+v_{3}\right) /\left(1-v_{2}\right)$ \\
\hline$\Psi \delta \Phi \zeta o$ & $-\left(d x_{1}+d x_{3}\right)$ & $d x_{2}$ & $-\left(v_{1}+v_{3}\right) /\left(1-v_{2}\right)$ \\
\hline$\Omega \in \Lambda \alpha O$ & $d x_{1}-d x_{2}$ & $-d x_{3}$ & $\left(v_{1}-v_{2}\right) /\left(1+v_{3}\right)$ \\
\hline$\Delta \beta \Phi \delta o$ & $-\left(d x_{1}-d x_{2}\right)$ & $-d x_{3}$ & $-\left(v_{1}-v_{2}\right) /\left(1+v_{3}\right)$ \\
\hline$\Omega \alpha \Xi \zeta o$ & $d x_{1}-d x_{3}$ & $-d x_{2}$ & $\left(v_{1}-v_{3}\right) /\left(1+v_{2}\right)$ \\
\hline$\Delta \delta \Theta \gamma O$ & $-\left(d x_{1}-d x_{3}\right)$ & $-d x_{2}$ & $-\left(v_{1}-v_{3}\right) /\left(1+v_{2}\right)$ \\
\hline$\Theta \gamma \Lambda \epsilon O$ & $d x_{3}-d x_{2}$ & $-d x_{1}$ & $\left(v_{3}-v_{2}\right) /\left(1+v_{1}\right)$ \\
\hline 30 & $-\left(d x_{3}-d x_{2}\right)$ & $-d x_{1}$ & $-\left(v_{3}-v_{2}\right) /\left(1+v_{1}\right)$ \\
\hline
\end{tabular}


This figure "Fig1.gif" is available in "gif" format from: http://arxiv.org/ps/gr-qc/9904081v1 
This figure "Fig2.gif" is available in "gif" format from: http://arxiv.org/ps/gr-qc/9904081v1 
This figure "Fig3.gif" is available in "gif" format from: http://arxiv.org/ps/gr-qc/9904081v1 
This figure "Fig4.gif" is available in "gif" format from: http://arxiv.org/ps/gr-qc/9904081v1 